\title{
Optical polarimetry, high-resolution spectroscopy and IR analysis of the Chamaeleon I dark cloud ${ }^{\star}$
}

\author{
E. Covino ${ }^{1}$, E. Palazzi ${ }^{2}$, B.E. Penprase ${ }^{3}$, H.E. Schwarz ${ }^{4}$, and L. Terranegra ${ }^{1}$ \\ 1 Osservatorio Astronomico di Capodimonte, Via Moiariello, 16, I-80131 Napoli, Italy \\ 2 ITESRE/C.N.R. Bologna, Italy \\ 3 Pomona College, Department of Physics and Astronomy, Claremont, CA, U.S.A. \\ 4 Nordic Optical Telescope, Apartado 474, E-38700 Sta. Cruz de La Palma, Canarias, Spain
}

Received March 21; accepted July 2, 1996

\begin{abstract}
We present optical polarimetry and high resolution spectroscopy of a sample of stars toward the Chamaeleon I dark cloud. We use our polarimetry which includes 33 stars to study the wavelength dependence of the degree and position angle of polarization.

From fits to the normalized wavelength dependence of interstellar polarization, we derive estimates of $\lambda_{\mathrm{Max}}$ ranging from $4500 \AA$ to $6700 \AA$, and $P_{\text {Max }}$ ranging from 3 to $8 \%$. The values of $\lambda_{\text {Max }}$ were found to be well correlated with the IRAS $100 \mu \mathrm{m}$ intensity, while $P_{\text {Max }}$ was found to increase with $E_{B-V}$.

High resolution spectra of the $\mathrm{Ca} \mathrm{II}, \mathrm{CH}$, and $\mathrm{CH}^{+}$ lines were obtained for 10 stars, which show two components of Ca II in absorption at $3.0<v_{\mathrm{LSR}}<5 \mathrm{~km} \mathrm{~s}^{-1}$, and $v_{\mathrm{LSR}}=-3.0 \mathrm{~km} \mathrm{~s}^{-1}$ and a single strong molecular $\mathrm{CH}$ absorption component at $3.0<v_{\mathrm{LSR}}<5.0 \mathrm{~km} \mathrm{~s}^{-1}$.

From our data we found, by interpretation of the various correlations between the polarimetry, photometry and IRAS fluxes, the following:

the probable presence of shocked molecular gas; a warm molecular $\mathrm{CH}$ component; small dust grains at the edges of the cloud, and larger grains in the central parts, which are causing the polarization.

Our results provide a consistent picture of the gas and dust content in the Cha I region, where larger grains, responsible of the starlight polarization, exist in the center of the cloud, surrounded by envelopes of warmer molecular and atomic material.
\end{abstract}

Key words: ISM: chamacleon clouds; dust, extinction; atoms; molecules — polarization — infrared: ISM: continuum

Send offprint requests to: E. Covino

* Based on observations collected at the European Southern Observatory at La Silla, Chile.

\section{Introduction}

The Chamaeleon I dark cloud $\left(\alpha \approx 11^{\mathrm{h}}, \delta \approx-76 \mathrm{deg}\right)$ is one of the nearest active star formation regions $(d \approx 120-150 \mathrm{pc})$. Thanks to its proximity and relatively high galactic latitude $\left(b \approx-16^{\circ}\right)$, this cloud is well suited for investigating the composition, magnetic field geometry and IR emission of a star forming cloud.

Observations of the Chamaeleon region were reviewed by Schwartz (1991), who provides a particularly complete account of the determination of the stellar content, extinction and distance toward the complex. The Cha I cloud was initially discovered to harbour an unusual number of $\mathrm{H} \alpha$ emitting stars (Henize 1954), and recent observations have determined that there are more than 100 association members (Prusti et al. 1991; Gauvin \& Strom 1992; Hartigan 1993).

Additional Young Stellar Object (YSO) members of Chamaeleon have been found from both X-ray emission from pointed ROSAT observations (Feigelson et al. 1993; Zinnecker et al. 1996) and deep IRAS (Assendorp et al. 1990) and $2 \mu \mathrm{m}$ observations of selected fields (Jones et al. 1985; Prusti et al. 1994). The consensus is that there are between 70 and 300 YSOs in Chamaeleon, in a cloud which is approximately 4 degrees across and has a mass of 700 to 1030 solar masses. Toriseva \& Mattila (1985) have estimated the dust to gas ratio in the cloud to be about 0.02 by mass. We should note that the inferred star formation efficiency of the Chamaeleon cloud is between $10 \%$ and $30 \%$ which is remarkably high.

While the stellar content of the Chamaeleon region is fairly well known, much uncertainty exists regarding the large scale structure of the ISM in the region. Early studies of extinction and distance to the cloud (Grasdalen et al. 1975; Thé et al. 1986) provided very different estimates of $R_{V}\left(3.1<R_{V}<5.3\right)$, and distance $d(115<d<215 \mathrm{pc})$. More recently, photometric observations (Franco 1991) suggest that the Chamaeleon region is contained in a sheet 
like cloud at $140 \mathrm{pc}$, which extends over several degrees on the sky into the constellation Musca. The maximum extinction in the Cha I cloud is thought to be in the range $4.0<A_{V}<6.0$, and the reddening of field stars from Vrba and Rydgren (1984) behind the cloud ranges from $0.05<E_{B-V}<1.27$, which completely samples the expected extinction in the Cha I cloud.

In this work we concentrate on the interstellar medium around the Chamaeleon I association, by combining new optical polarimetry and optical spectroscopy of atomic and molecular absorption features with IR data.

Recent work on the polarimetry of the Chamaeleon region has also been published (Whittet et al. 1994; McGregor et al. 1994) as has a comparison between IR and UV emission and extinction (Boulanger et al. 1994). Our work seeks to provide a unified approach in which the different observations provide a complementary picture about the interrelations between dust and gas in the Chamaeleon cloud.

Where possible we will analyze the connections between the observed optical polarization, IR emission and optical absorption lines for the stars in the Chamaeleon region. Photometry suggests a very low foreground reddening $\left(E_{B-V} \approx 0.04\right.$ is reported by Whittet et al. 1987, and an even smaller value of $E_{B-V} \approx 0.007$ is found by Franco 1991), which insures that optical absorption lines and the polarization of background stars of moderate distances are due to the Cha I dark cloud, and are not significantly affected by foreground absorption.

\section{Observations}

\subsection{Optical polarimetry}

Our sample of 33 background stars was selected from the list of field stars provided by Whittet et al. (1987). Linear polarization measurements were carried out in $1992 \mathrm{March}$ 13 to 17 at the ESO/MPI $2.2 \mathrm{~m}$ telescope equipped with the two-channel photo-polarimeter PISCO (Stahl et al. 1986; Schwarz 1989). Measurements were obtained in the Cousins $U B V R I$ system using a diaphragm of 15 arcsec. The instrumental polarization compensation mode, involving a rotation of a compensating phase plate unit by 180 degrees, was systematically used. The data acquisition was performed using the two-apertures mode for sky polarization compensation (the two apertures being separated by $66^{\prime \prime}$ in the East-West direction). Calibration in polarization, $P$, and position angle, $\theta$, was done through observations of the polarization standard star HD 147084 (o Sco) (Hsu \& Breger 1982).

The uncertainty in the position angle in all cases was estimated to be less than 2 degrees, using polarized standard stars and the internal calibration of the polarimeter. The instrumental polarization was measured by observing the unpolarized standard stars HD 100623, HD 115617 (61 Vir) and HR 6060 (18 Sco) (Serkowski 1974;
Walborn 1968). Measurements of some bright stars were also performed with a polaroid inserted in order to determine the instrumental efficiency. These yielded negligible corrections.

The reduction of the polarimetric data was performed using a revised version of the MIDAS procedure PISCO. For each star the degree of polarization was determined, with values ranging from zero up to about 8 percent. The observational errors are determined from the power in the 5th and higher harmonics in the Fourier-transform, using an algorithm which is part of the PISCO data reduction package (Schwarz 1989). Also, the correction for noise biasing, according to the analytical formula $p=p^{\prime}\left[1-\left(\sigma / p^{\prime}\right)^{2}\right]^{1 / 2}$ has been applied to the observed polarization, $p^{\prime}$, using the estimated standard deviation of the polarization, $\sigma$, derived from the PISCO reduction procedure (Clarke \& Stewart 1986).

The list of observed stars is given in Table 1, together with other relevant information. The optical polarization data are given in Table 2. For reference, the first column of each table gives the list number from Whittet et al. (1987).

\subsection{High resolution spectroscopy}

The background stars earlier than A0, and suitable for the spectroscopic study of the cloud were selected from the polarimetry sample. In addition, we included the stars HD 97300 and HD 97048, which are thought to be members of the Cha I association, to improve the completeness of our spectroscopic sample.

High resolution spectroscopy was done with the Coudé Auxiliary Telescope (CAT) of the European Southern Observatory at Cerro La Silla (Chile) during two observing runs in 1992 March and 1994 January. The data were obtained using an RCA CCD (ESO \# 9) and the CES short camera in the blue path. This instrument configuration provided a resolving power, $\lambda / \Delta \lambda>70000$ at the studied wavelengths. A few additional spectra were obtained in 1994 July using the CAT with the CES long camera, operated in remote control from Garching. The July data have a higher resolving power $\lambda / \Delta \lambda=130000$, and were used to complete coverage of the region, and obtain more detailed velocity profiles for selected stars.

Data reduction was performed using the IRAF system, in which the standard CCD reduction packages were used to remove cosmic rays, perform bias subtraction and flatfield the images. Spectral extraction was done using the optimal extraction algorithm in the IRAF task apsum, in which pixel values across an order of a spectrum are weighted according to the calculated signal to noise ratio. The signal to noise was estimated using the readout noise and gain of the CCD. Spectra were wavelength calibrated using a Thorium/Argon calibration lamp, and a linear interpolation of matched features. 
Table 1. Stellar parameters

\begin{tabular}{|c|c|c|c|c|c|}
\hline F\# & ID & Sp.T. & $\bar{V}$ & $E(B-V)$ & Notes \\
\hline F1 & VR k & K4III & 10.31 & 0.13 & \\
\hline $\mathrm{F} 2$ & VR g & B8V & 9.90 & 0.62 & \\
\hline F3 & HD 94414 & $\mathrm{~B} 2 \mathrm{~V}$ & 7.99 & 0.70 & VR e \\
\hline F6 & VR d & $\mathrm{A} 2 \mathrm{~V}$ & 11.06 & 0.58 & \\
\hline F7 & VR c & B5V & 10.30 & 0.58 & \\
\hline F9 & $\mathrm{VR} \mathrm{j}$ & KOIII & 9.58 & 0.61 & IRAS10577-7649 \\
\hline F10 & Glass k & K3III & 13.74 & 0.77 & \\
\hline F11 & $\mathrm{VR} \mathrm{b}$ & B9V & 10.47 & 0.80 & \\
\hline F16 & Glass C & G2IV & 11.46 & 0.81 & CHXR11 \\
\hline F18 & Glass 1 & G8III & 15.22 & 1.27 & \\
\hline F 21 & Glass T & K3III & 11.39 & 0.68 & \\
\hline F 22 & Glass D & G8III & 14.10 & 1.03 & \\
\hline F 23 & Glass E & M5III & 12.93 & 0.90 & \\
\hline $\mathrm{F} 24$ & HD 96675 & $\mathrm{~B} 7 \mathrm{~V}$ & 7.67 & 0.31 & VR a, CHXR16, IRAS11042-7552 \\
\hline F 25 & Glass B & G8III & 13.23 & 1.27 & \\
\hline F28 & Glass G & K4III & 15.14 & 1.10 & \\
\hline F29 & Glass $\mathrm{h}$ & $\mathrm{K} 4 \mathrm{~V}$ & 13.33 & 0.31 & CHXR28, T-assoc. meber \\
\hline F30 & Glass A & K3III & 11.47 & 0.46 & \\
\hline F33 & Glass O & G8III & 15.09 & 1.05 & \\
\hline F34 & Glass Q & K3V & 14.32 & 0.53 & CHXR47, IRAS11091-7716, T-assoc. meber \\
\hline F36 & Glass P & KOIII & 13.76 & 1.22 & \\
\hline F37 & VR h & G9III & 9.56 & 0.31 & \\
\hline F39 & VR i & K3III & 10.07 & 0.32 & \\
\hline F40 & HD 98143 & B8III & 7.57 & 0.65 & VR f \\
\hline F41 & HD 98294 & $\mathrm{~B} 8 \mathrm{~V}$ & 8.52 & 0.26 & \\
\hline F42 & HD 98561 & A3.IV & 8.33 & 0.34 & \\
\hline F43 & HD 98627 & K5III & 7.38 & 0.25 & \\
\hline F44 & HD 98672 & A5V & 6.26 & 0.00 & \\
\hline F47 & HD 99015 & A5-IV & 6.42 & 0.05 & \\
\hline F48 & HD 99161 & B9.5V & 8.87 & 0.18 & \\
\hline F50 & HD 99515 & A8IV & 9.37 & 0.13 & \\
\hline F52 & HD 99759 & B9.5V & 8.48 & 0.30 & \\
\hline F54 & HD 100401 & G6-IV & 8.36 & 0.28 & \\
\hline T32 & HD 97048 & $\mathrm{~A} 0 \mathrm{~V}$ & 8.45 & $0.39 *$ & Ced 111 \\
\hline $\mathrm{T} 41$ & HD 97300 & B9V & 8.90 & $0.46 *$ & Ced 112 \\
\hline
\end{tabular}

* from Rydgren A.E., 1980, AJ 85, 444.

Table 2. Polarization data

\begin{tabular}{|c|c|c|c|c|c|c|c|c|c|c|c|c|c|c|c|c|c|c|c|c|c|c|}
\hline \multirow{2}{*}{$\begin{array}{l}\overrightarrow{\mathrm{F}} \\
\mathrm{F} 1\end{array}$} & \multicolumn{2}{|c|}{$(\% U)$} & \multicolumn{2}{|c|}{$(\% B)$} & \multicolumn{2}{|c|}{$(\% V)$} & \multicolumn{2}{|c|}{$(\% R)$} & \multicolumn{2}{|c|}{$(\% \mathrm{I})$} & \multicolumn{2}{|c|}{$\theta(U)$} & \multicolumn{2}{|c|}{$\theta(B)$} & \multicolumn{2}{|c|}{$\theta(V)$} & \multicolumn{2}{|c|}{$\theta(R)$} & \multicolumn{2}{|c|}{$\theta(I)$} & \multirow{2}{*}{$\begin{array}{l}P_{\text {Max }} \\
3.46\end{array}$} & \multirow{2}{*}{$\begin{array}{r}\lambda_{\operatorname{Max}} \\
0.54\end{array}$} \\
\hline & 2.90 & 0.03 & $3.33 \pm$ & 0.15 & 3.38 & $=0.09$ & $3.23=$ & \pm 0.06 & $3.07 \pm$ & 0.06 & $115.07=$ & \pm 8.72 & $117.53=$ & \pm 1.26 & $117.89=$ & \pm 0.76 & $117.57 \pm$ & 0.56 & $118.19=$ & \pm 0.53 & & \\
\hline $\mathrm{F} 2$ & 2.41 & 0.52 & 3.26 & 0.14 & 3.80 & 0.10 & 3.89 & 0.07 & 3.74 & 0.06 & 123.30 & 5.98 & 131.72 & 1.25 & 132.52 & 0.77 & 133.65 & 0.52 & 134.13 & 0.48 & 3.91 & 0.67 \\
\hline F3 & 3.83 & 0.17 & 4.31 & 0.10 & 4.81 & 0.06 & 4.98 & 0.04 & 4.79 & 0.04 & 112.97 & 1.27 & 120.29 & 0.65 & 120.14 & 0.38 & 121.12 & 0.21 & 122.18 & 0.24 & 5.03 & 0.61 \\
\hline$F_{6}$ & 4.71 & 0.66 & 4.93 & 0.19 & 5.39 & 0.11 & 5.13 & 0.09 & 4.85 & 0.11 & 114.08 & 3.98 & $11 \overline{7} .64$ & 1.09 & 116.95 & 0.60 & 116.34 & 0.48 & 117.37 & 0.68 & 5.54 & 0.55 \\
\hline F7 & 4.81 & 0.23 & 5.39 & 0.11 & 5.79 & 0.09 & 5.69 & 0.09 & 5.22 & 0.06 & 110.04 & 1.36 & 117.50 & 0.57 & 116.75 & 0.44 & 117.08 & 0.43 & 118.24 & 0.36 & 5.89 & 0.56 \\
\hline Fe & 3.95 & 0.64 & 3.95 & 0.20 & 4.61 & 0.09 & 4.66 & 0.06 & 4.47 & 0.07 & 131.08 & 4.60 & 125.75 & 1.44 & 127.96 & 0.54 & 127.99 & 0.37 & 128.37 & 0.43 & 4.76 & 0.58 \\
\hline F10 & - & & 4.13 & 1.96 & 3.87 & 0.38 & 4.13 & 0.23 & 3.87 & 0.14 & - & - & 144.33 & 12.30 & 121.17 & 2.80 & 119.73 & 1.57 & 120.53 & 1.01 & 4.06 & 0.65 \\
\hline F11 & 3.43 & 0.76 & 4.30 & 0.22 & 4.75 & 0.14 & 4.43 & 0.11 & 4.16 & 0.12 & 103.82 & 6.17 & 115.37 & 1.49 & 115.21 & 0.86 & 113.50 & 0.71 & 113.36 & 0.82 & 4.60 & 0.59 \\
\hline F16 & 4.91 & 1.54 & 5.94 & 0.46 & 6.61 & 0.13 & 6.97 & 0.07 & 6.68 & 0.04 & 103.78 & 8.57 & 121.84 & 2.22 & 122.26 & 0.58 & 122.38 & 0.30 & 123.57 & 0.17 & 6.98 & 0.63 \\
\hline F18 & & & & & & - & 3.52 & 0.57 & 3.74 & 0.31 & - & - & 92.67 & 36.14 & 135.43 & 20.90 & 137.15 & 4.56 & 135.71 & 2.36 & - & \\
\hline F21 & 3.85 & 2.50 & 4.98 & 0.60 & 5.64 & 0.25 & 5.64 & 0.13 & 4.91 & 0.10 & 116.11 & 15.62 & 118.69 & 3.42 & 117.59 & 1.11 & 117.04 & 0.68 & 117.30 & 0.60 & 5.78 & 0.57 \\
\hline F 22 & - & - & 10.92 & 5.98 & 7.11 & 0.68 & 7.39 & 0.25 & 7.11 & 0.19 & - & - & 126.56 & 13.76 & 119.24 & 2.82 & 118.79 & 0.97 & 119.39 & 0.77 & 7.33 & 0.69 \\
\hline F23 & 2.38 & 3.92 & 6.72 & 1.95 & 6.94 & 0.36 & 7.25 & 0.09 & 6.94 & 0.06 & 142.21 & 24.50 & 114.84 & 7.99 & 116.00 & 1.48 & 117.04 & 0.36 & 116.68 & 0.23 & 7.20 & 0.67 \\
\hline F24 & 2.48 & 0.08 & 2.86 & 0.07 & 2.98 & 0.18 & 3.34 & 0.29 & 2.68 & 0.03 & 119.74 & 1.05 & 127.58 & 0.70 & 128.08 & 1.73 & 128.99 & 2.51 & 129.30 & 0.34 & 3.14 & 0.57 \\
\hline F25 & 7.12 & 5.68 & 6.66 & 1.29 & 7.99 & 0.32 & 7.99 & 0.14 & 7.46 & 0.09 & 163.09 & 17.87 & 125.19 & 5.46 & 123.52 & 1.18 & 123.45 & 0.50 & 123.44 & 0.34 & 7.91 & 0.84 \\
\hline F28 & - & - & - & & & - & 7.09 & 0.46 & 7.08 & 0.18 & - & - & - & - & - & - & 139.18 & 1.87 & 138.22 & 0.67 & - & - \\
\hline F29 & - & $\rightarrow$ & 4.57 & 1.74 & 4.80 & 0.60 & 4.88 & 0.18 & 4.80 & 0.13 & - & - & 136.61 & 10.19 & 148.36 & 3.64 & 149.69 & 1.07 & 150.47 & 0.77 & 4.92 & 0.68 \\
\hline F30 & 3.88 & 1.59 & 3.93 & 0.62 & 5.44 & 0.49 & 3.01 & 0.38 & 3.02 & 0.51 & 93.29 & 10.86 & 115.21 & 4.45 & 110.69 & 2.59 & 108.19 & 3.62 & 104.45 & 4.7 & 4.24 & 0.45 \\
\hline F33 & - & - & - & & 3.75 & 0.94 & 4.01 & 0.49 & 3.75 & 0.25 & - & - & - & - & 159.46 & 13.81 & 148.84 & 3.46 & 150.44 & 1.89 & 7.67 & - \\
\hline F34 & - & - & 17.12 & 6.72 & 8.22 & 0.66 & 8.84 & 0.26 & 8.22 & 0.18 & - & - & 141.85 & 10.47 & 131.14 & 2.10 & 135.80 & 0.83 & 133.71 & 0.57 & 8.97 & 0.60 \\
\hline F 36 & - & - & 11.79 & 3.03 & 1.70 & 0.61 & 3.83 & 10.57 & 1.70 & 0.10 & - & - & 134.23 & 7.13 & 135.09 & 1.55 & 136.96 & 4.1 & 134.00 & 0.25 & 8.58 & 0.81 \\
\hline F37 & 2.42 & 0.58 & 3.14 & 0.15 & 3.13 & 0.08 & 3.13 & 0.05 & 2.86 & 0.05 & 113.26 & 6.70 & 119.72 & 1.39 & 119.52 & 0.74 & 118.39 & 0.43 & 119.44 & 0.46 & 3.21 & 0.57 \\
\hline F39 & 2.69 & 0.88 & 3.22 & 0.16 & 3.19 & 0.05 & 3.03 & 0.04 & 2.70 & 0.03 & 119.37 & 8.85 & 127.14 & 1.38 & 126.07 & 0.4 & 126 & 0.4 & 127.04 & 0.3 & 3.24 & 0.53 \\
\hline F 40 & 5.74 & 0.19 & 6.88 & 0.14 & 7.69 & 0.33 & 7.64 & 0.06 & 6.86 & 0.03 & 125.48 & 0.95 & 131.20 & 0.60 & 131.06 & 1.23 & 131.43 & 0.24 & 131.82 & 0.13 & 7.62 & 0.59 \\
\hline F 41 & 2.12 & 0.19 & 2.38 & 0.07 & 2.58 & 0.06 & 2.61 & 0.04 & 2.41 & 0.05 & 110.24 & 2.61 & 119.87 & 0.84 & 120.64 & 0.64 & 120.37 & 0.41 & 121.69 & 0.64 & 2.66 & 0.58 \\
\hline F 42 & 2.11 & 0.17 & 2.68 & 0.06 & 2.80 & 0.33 & 2.80 & 0.03 & 2.61 & 0.04 & 115.28 & 2.30 & 120.14 & 0.65 & 121.50 & 3.3 & 120.87 & 0.30 & 122.27 & 0.4 & 2.85 & 0.59 \\
\hline F43 & - & - & 2.07 & 0.11 & 2.34 & 0.72 & 2.34 & 0.13 & 2.62 & 0.10 & & & 121.49 & 1.58 & 119.39 & 8.71 & 125.91 & 1.58 & 123.77 & 1.06 & 2.51 & 0.70 \\
\hline F44 & 0.47 & 0.06 & & - & - & - & 0.16 & 0.04 & 0.19 & 0.02 & 86.96 & 3.62 & - & - & - & - & 71.38 & 6.56 & 66.66 & 2.81 & - & - \\
\hline F 47 & 0.59 & 0.15 & 0.14 & 0.08 & - & - & - & - & 0.13 & 0.10 & 76.43 & 7.29 & 85.26 & 14.64 & - & - & - & - & 146.94 & 17.91 & - & - \\
\hline F48 & 1.82 & 0.28 & 2.13 & 0.08 & 2.26 & 0.07 & 2.27 & 0.07 & 2.09 & 0.07 & 113.62 & 4.42 & 123.85 & 1.12 & 126.03 & 0.89 & 127.62 & 0.93 & 127.46 & 0.95 & 2.31 & 0.57 \\
\hline F50 & 1.46 & 0.46 & 1.44 & 0.1 & 1.59 & 0.07 & 1.66 & 0.08 & 1.41 & 0.0 & 130.18 & 8.65 & 139.87 & 2. & 138.26 & 1.3 & 137.66 & 1.30 & 140.50 & 1.61 & 1.64 & 0.54 \\
\hline F52 & 2.32 & 0.20 & 2.66 & 0.08 & 2.97 & 0.06 & 2.97 & 0.05 & 2.82 & 0.06 & 119.37 & 2.42 & 128.30 & 0.89 & 128.86 & 0.55 & 129.19 & 0.43 & 129.84 & 0.58 & 3.03 & 0.60 \\
\hline F54 & 2.23 & 0.37 & 2.64 & 0.07 & 2.68 & 0.05 & 2.59 & 0.03 & 2.37 & 0.03 & 118.26 & 4.71 & 125.51 & 0.75 & 125.38 & 0.49 & 126.01 & 0.34 & 125.37 & 0.38 & 2.72 & 0.54 \\
\hline
\end{tabular}


For each spectrum a fit to the continuum was performed using a high order spline, and a correction was applied to transform the spectra to a uniform LSR velocity reference frame, by removing the velocity contributed by terrestrial and solar motions. Column densities were computed by using a Voigt profile model, based on a version of the STARLINK curve of growth programme adapted for IRAF. For some of the spectra, line profiles were modeled using the MIDAS image processing package. Each line profile was modeled using the minimum number of components necessary to reproduce the observed data with residual values comparable to the noise in the data. Our spectral resolution limited us to separating line components with velocity differences of more than $1-2 \mathrm{~km} \mathrm{~s}^{-1}$, and we cannot rule out the possibility of very narrow blended components. However, since the turbulent velocities in molecular clouds are expected to be $1-2 \mathrm{~km} \mathrm{~s}^{-1}$, we believe our instrumental resolution is able to resolve the components present in the Chamaeleon cloud. This is also consistent with the determinations based on $\mathrm{CO}$ observations in the Cha I cloud obtained by Dubath et al. (1995).

\subsection{IR data analysis}

For the Chamaeleon region we obtained the latest coadded plates for all four bands of the ISSA survey. The pixel size within the plates is $1.5^{\prime}$ which, in the case of the $100 \mu \mathrm{m}$ band, oversamples the actual instrumental resolution of the satellite, which is closer to $4^{\prime}$. We removed zodiacal background residuals from each plate by using an iterative background removal programme which selects background points based on an analysis of the histogram of pixel values, and fits a flat background to the selected points. IRAS colors were derived using the programme skyview from IPAC, which averages pixel fluxes in a 9 pixel region centered on the coordinates of our program stars. The larger sampling region makes that the IRAS colours have a higher signal to noise ratio, and samples a region $4.5^{\prime \prime}$ square, with uncertainties in the IRAS flux, $F_{\lambda}$, of $\delta_{\lambda}<0.08 \mathrm{mJy} \mathrm{Sr}^{-1}$ for $12 \mu \mathrm{m}$ and $25 \mu \mathrm{m}$, and $\delta_{\lambda}<0.15 \mathrm{mJy} \mathrm{Sr}^{-1}$ for the $60 \mu \mathrm{m}$ and $100 \mu \mathrm{m}$ passbands.

\section{Results and discussion}

\subsection{Polarimetry}

Table 1 presents the stellar parameters for our sample. Earlier photometric and polarimetric work in the Chamaeleon region has analyzed the stellar content of both member and field stars (Whittet et al. 1987), and a search of the SIMBAD database and reading of literature has produced the notes included in Table 1. Important in the discussion of any polarimetry is the possibility of intrinsic polarization from the source. We chose to observe stars which were optically determined to be field stars, which are expected to have little or no intrinsic polarization. Three of the stars in our sample are IRAS point sources, which is probably a result of the late spectral type and bright magnitude. Recent pointed ROSAT observations (Feigelson et al. 1993; Zinnecker et al. 1996), however, have identified four new YSOs which are associated with our "field" stars, these are indicated in Table 1. Two of them are both IRAS point sources and X-ray sources. The polarimetry and the IR emission of these stars will probably be influenced by circumstellar material.

Figure 1 shows an IRAS $100 \mu$ image of the Chamaeleon I cloud, with the locations of our program stars indicated.

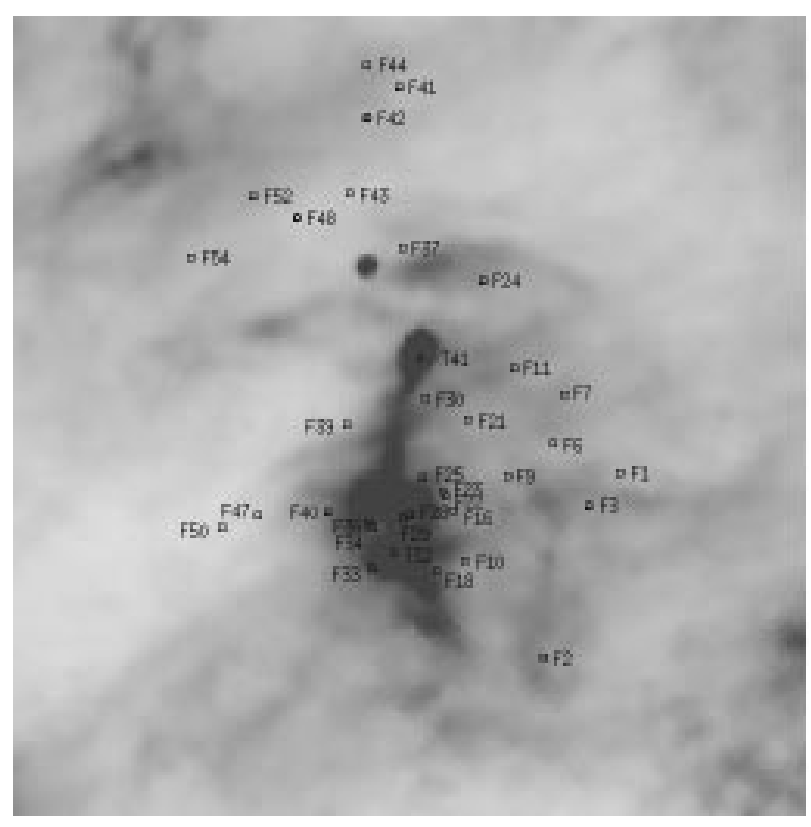

Fig. 1. Location of program stars plotted on IRAS $100 \mu \mathrm{m}$ map of the Cha I cloud

Measurements of optical linear polarization of starlight can be used to trace the geometry of the interstellar magnetic field component, $\boldsymbol{B}_{\perp}$, in the plane of the sky. The observed polarization is produced by the differential extinction by non-spherical dust grains associated with the interstellar clouds along the line of sight. It is assumed in fact that elongated paramagnetic dust grains are aligned by the local interstellar magnetic field, via the DavisGreenstein mechanism with their short axes parallel to the field direction. Strong evidence that extinction by dust grains is responsible for the observed polarization comes from the existence of a correlation between the degree of polarization and the amount of reddening (Spitzer 1978).

In Fig. 2 we present a plot for the Chamaeleon I cloud, with the observed polarization vectors for our program stars, superimposed onto a plot of IRAS $100 \mu \mathrm{m}$ contours, with equatorial coordinates labeled. The magnetic field 
vectors would be expected to follow the polarization vectors, and they are approximately parallel to the galactic plane. The dispersion in the values of the polarization angle is very small, typically 2 degrees or less. The complete results of the polarization measurements are presented in Table 2 for our sample of stars in the five passbands.

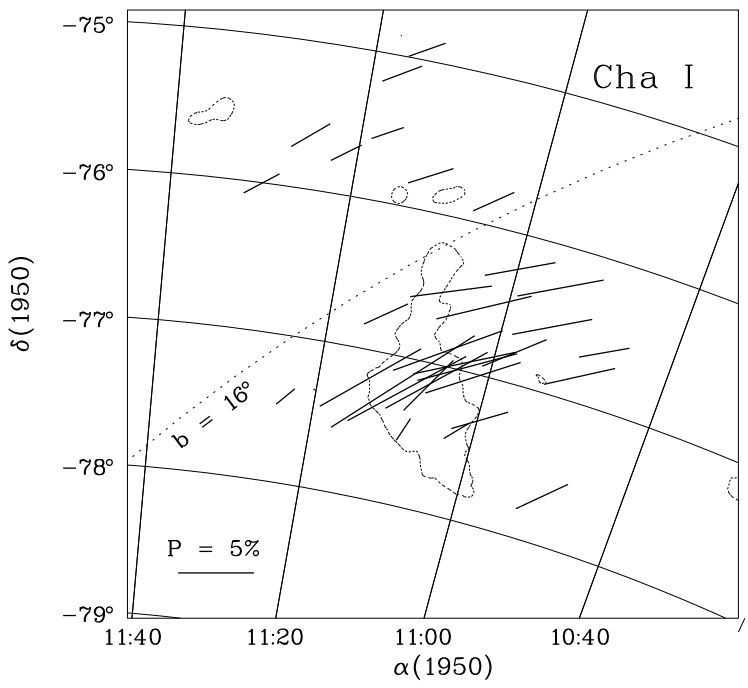

Fig. 2. Average polarization vectors for polarimetric program stars. The $100 \mu \mathrm{m}$ contours of IRAS are also included, as are coordinate grids for equatorial coordinates and galactic plane parallel. The length of the symbol is proportional to the percentage polarization

We have adopted the parameterisation of the wavelength dependence of the linear polarization, referred to as Serkowski's model (Coyne et al. 1974),

$$
P(\lambda) / P_{\operatorname{Max}}=\exp \left[-K \ln ^{2}\left(\lambda_{\operatorname{Max}} / \lambda\right)\right]
$$

where $\lambda_{\mathrm{Max}}$ is the wavelength at which the maximum polarization, $P_{\mathrm{Max}}$, is observed, and $K \approx 1.15$. The value of $\lambda_{\text {Max }}$ for our sample ranged from 0.35 to $0.9 \mu \mathrm{m}$ and typically was found to be near $\lambda_{\operatorname{Max}}=0.55 \mu \mathrm{m}$. Larger values of $\lambda_{\mathrm{Max}}$ are expected in direction of dense clouds, indicating an increase in the mean grain size. A discussion of the theoretical arguments behind the Serkowski polarization model may be found in Spitzer (1978).

For selected stars we have fitted the percentage polarization with a Serkowski polarization model to derive values of $\lambda_{\mathrm{Max}}$ and $\mathrm{P}_{\mathrm{Max}}$. The data points, and their best fits are presented in Fig. 3. The Serkowski model was fitted only to those stars which had accurate polarization measurements in at least four passbands. We excluded measurements from our fit which had polarization uncertainties $P_{\lambda}>1.6 \%$.

To check the effect of fitting the Serkowski model with four points, we also did all the fits using both 5 and 4

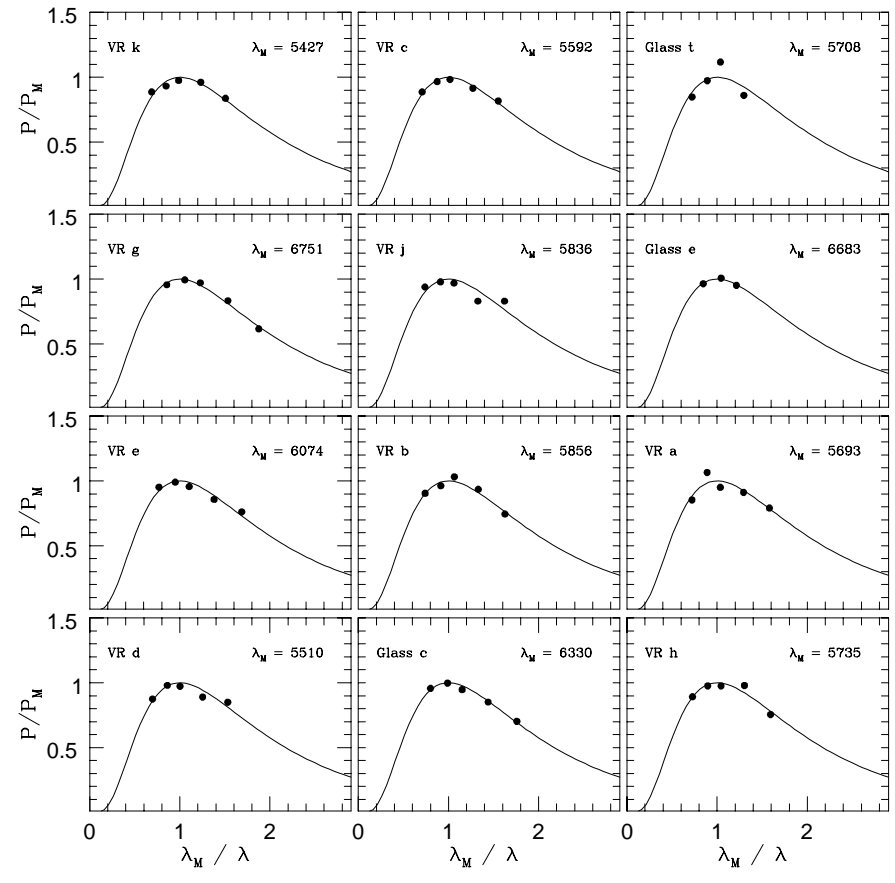

Fig. 3. a) Least square fits to Serkowski's law for individual stars. The Serkowski polarization model was fitted with $K=1.15$, and free parameters $P_{\mathrm{Max}}$ and $\lambda_{\mathrm{Max}}$

points and, typically, the values of $\lambda_{\mathrm{Max}}$ derived in the two ways do not differ by more than $100 \AA$. For 19 stars we have fitted the Serkowski polarization model with 5 points, while 9 stars were done with 4 points, since the late spectral types of the stars prevented accurate measurements of $U$ band polarization. Seven stars were not fitted at all, since both the $U$ and $B$ polarization were found to be inaccurate, or the star was found to be unpolarized.

In Fig. 4 we present plots of the percentage polarization for each of the five bands against the color excess $E_{B-V}$. It is clear that the polarization rises for $E_{B-V}<0.5$ at a different slope than for the largest values of $E_{B-V}>0.5$. A similar change in slope has been observed by Whittet et al. (1991), and it is believed to reflect a decrease in the efficiency of polarization at the highest extinctions due to either more spherical grains, or de-alignment of the grains in the densest parts of the sight lines. Figure 5 shows the maximum polarization $P_{\mathrm{Max}}$ of each star, plotted against the color excess $E_{B-V}$. Also shown in Fig. 5 are separate fits for the sight lines where 4 or 5 points were used to fit the data to the Serkowski model. We see a gradually increasing function, with a fair amount of dispersion in the measured values of $P_{\mathrm{Max}}$.

From the parameterisation of the polarization wavelength dependence it is also possible to determine $\lambda_{\mathrm{Max}}$, and we examined the behaviour of $\lambda_{\mathrm{Max}}$ as a function of extinction. The values of $\lambda_{\text {Max }}$ increase with $E_{B-V}$ and a plot is presented in Fig. 6 for our sample. The increase in 


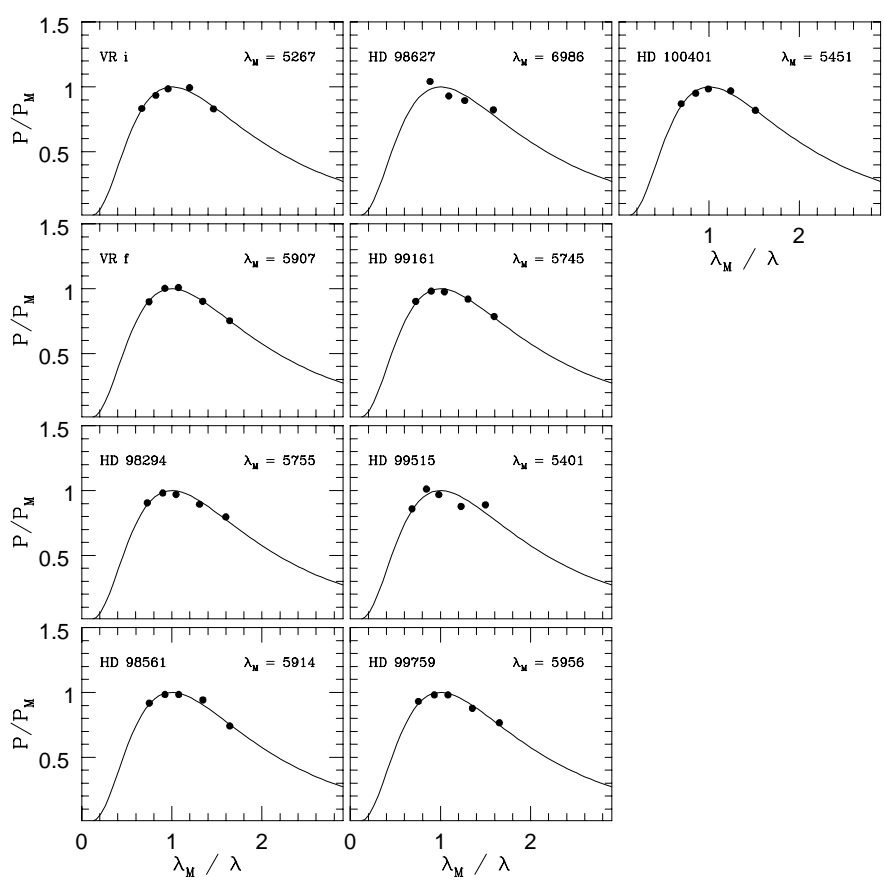

Fig. 3. b) Same as in Fig. 3a

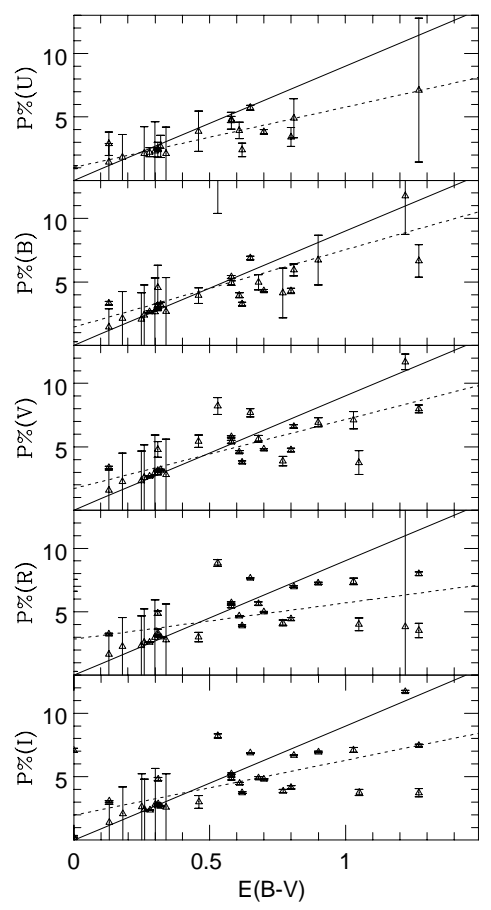

Fig. 4. Polarization percentage for each of the five passbands for the polarimetric sample. Included are the best fits to the data (dashed line) and "maximum" polarization, $\left(P / E_{B-V}\right)_{\text {Max }}=9.0 \% \mathrm{mag}^{-1},($ solid line) from Spitzer (1978). The different slope between high and low extinction sightlines is apparent

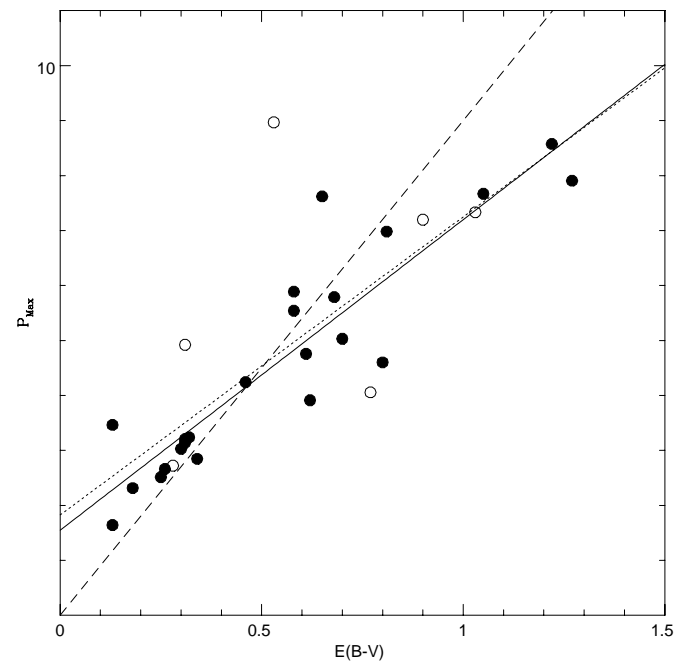

Fig. 5. Maximum polarization, $P_{\mathrm{Max}}$, plotted against $E_{B-V}$ for the polarimetric sample. The filled circles indicate sightlines which had 5 measurements for fitting to the Serkowski model, while the open circles are sightlines which only had measurements in 4 filters. The dotted line shows the best fit to all of the points, and the solid line the best fit to sightlines which included 5 measurements. The dashed line represents the "maximum" polarization efficiency from Spitzer (1978)

$\lambda_{M a x}$ at large $E_{B-V}$ is consistent with larger dust grains occurring in denser sight lines with high extinction, perhaps as the result of ices accumulating on the grains.

In Fig. 7 we present a plot of the distribution of $\lambda_{\text {Max }}$, which appears to be bimodal, with a smaller population of points having larger values of $\lambda_{\text {Max }}$. It appears that most of the large values of $\lambda_{\text {Max }}$ also coincide with the peak $100 \mu \mathrm{m}$ emission from the cloud, based on an examination of the location of the program stars on the IRAS map of Fig. 1.

Variations in $\lambda_{\operatorname{Max}}$ are generally attributed to variations in the mean grain size toward the region. Some complications can arise, however, in regions with multiple clouds along the line of sight, which may have different values of $\lambda_{\mathrm{Max}}$, or different orientations of the mean magnetic field. In the former case, significant deviations from the Serkowski model may be found, while in the latter case, the electric field vector orientation may be a function of wavelength. Clarke and Al-Roubaie (1984) have modeled the effects of various grain size distributions, and specifically the rotation of the polarization vector $\theta_{I}-\theta_{B}$ with the angle $\phi$ between two clouds along the sight line. Additionally a linear relation exists between the width parameter $K$ and $\lambda_{\text {Max }}$. The physical significance of the parameter $K$ from the Serkowski model has been examined by Whittet et al. (1991), but is presently uncertain whether $K$ varies significantly with direction within the Galaxy.

We have assumed a value of 1.15 for the Serkowski $K$ parameter, and also that most of the polarization 


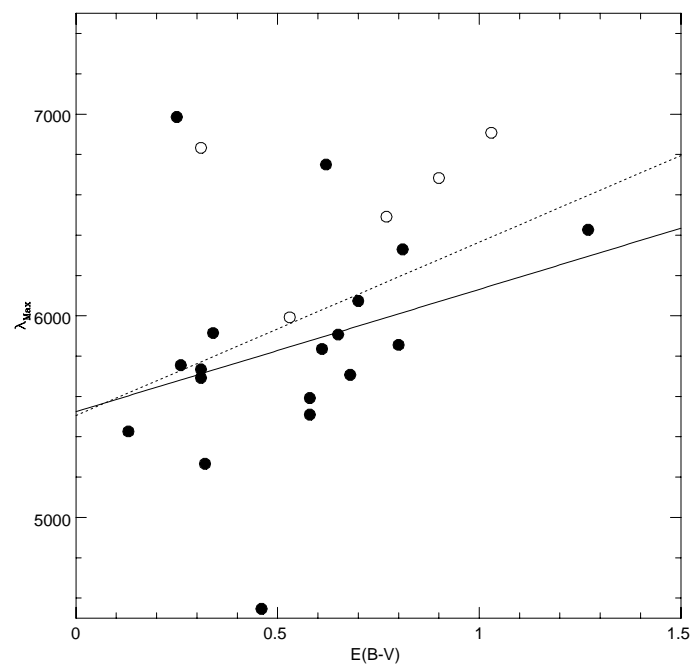

Fig. 6. Wavelength of maximum polarization, $\lambda_{\mathrm{Max}}$ versus $E_{B-V}$. The dotted line represents the least square fits to all data points, while the solid line gives the least square fits for only values of $\lambda_{\text {Max }}$ derived from more than three points fits to the Serkowski's law (filled circles)

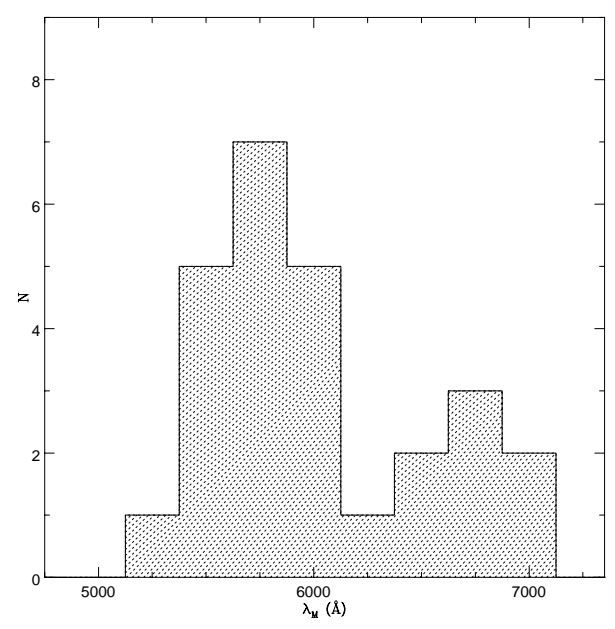

Fig. 7. Histogram of $\lambda_{\mathrm{Max}}$ values, showing a bimodal population with the larger values of $\lambda_{\mathrm{Max}}$ concentrated mostly in the center of the cloud

results from a single component. The spectroscopic results of Sect. 3.3 suggest that there are two components of the ISM in the line of sight to the Cha I association. The second component, which appears in several of the Ca II spectra, is much weaker, however, and therefore our assumption of a single dominant component is valid. Our observed rotation of the polarization angles for a given sight line varied by less than $2^{\circ}$ for nearly all sight lines, which is also consistent with a single dominant component of polarizing interstellar medium (Clarke \& Al-Roubaie 1984).

\subsection{Analysis of IRAS infrared colors}

Infrared observations of nearby molecular clouds and atomic cirrus have also been used to deduce an increased small grain content from increased $R(12,100)$, and decreased $I_{100 \mu}$ near the edges of clouds, which undergo rapid fluctuations of the number of small grains attributed variously to condensations from gas, removal from larger grains, and from slight redistributions between small $(a<30 \AA)$ and mid-sized $(30<a<100 \AA)$ grains (Désert et al. 1990; Bernard \& Boulanger 1993).

We have analyzed the values of $R(12,100)$ and other IRAS colours, and present the results for our program star sight lines in Table 3 . The IRAS colours were also examined for systematic trends with some of our other polarimetric and spectroscopically observed quantities. Figure 8 presents a plot of percentage polarization against $R(12,100)$. A clear decrease in polarization is seen as $R(12,100)$ increases, suggesting that the processes which disrupt the grains in the cloud to produce the smaller particles, also are capable of either realigning or destroying the larger grains responsible for optical polarization. The fact that in Fig. 9 (top) we see little or no correlation between $\lambda_{\text {Max }}$ and $R(12,100)$ suggests that the process of creating the small grain population minimally affects the grain size distribution of the polarizing grains, and therefore the small grains producing the enhanced values of $R(12,100)$ are probably an independent population from the polarizing grains.

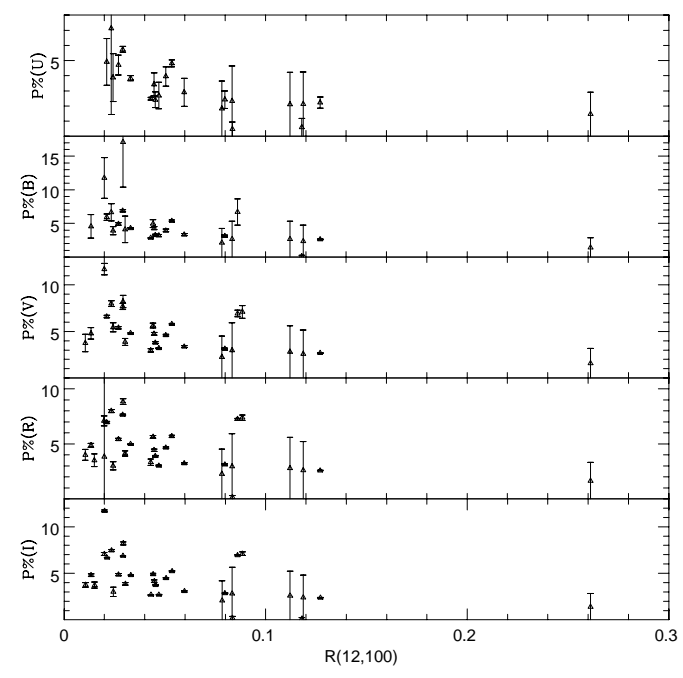

Fig. 8. Plot of percentage polarization against $R(12,100)$ infrared color

Bernard \& Boulanger (1993) have modeled the various contributions of PAH and other small molecules to the small grain content, and Désert et al. (1990) have determined expected IR colours with mixtures of PAH's and small grains. Boulanger et al. (1994) have determined that 
Table 3. Infrared data from IRAS. The $100 \mu$ IR fluxes, $I(100)$, are in $\mathrm{mJy} \mathrm{Sr}^{-1}$

\begin{tabular}{|c|c|c|c|c|c|c|c|c|}
\hline F\# & ID & $E(B-V)$ & $R_{v}$ & $A_{v}$ & $I(100)$ & $R(12,100)$ & $R(12,25)$ & $R(60,100)$ \\
\hline F1 & VR k & 0.13 & 3.22 & 0.42 & 5.87 & 0.06 & 0.85 & 0.15 \\
\hline $\mathrm{F} 2$ & VR g & 0.62 & 4.01 & 2.49 & 10.19 & 0.04 & 0.83 & 0.15 \\
\hline F3 & HD 94414 & 0.70 & 3.61 & 2.52 & 9.27 & 0.03 & 0.73 & 0.11 \\
\hline F6 & VR d & 0.58 & 3.27 & 1.90 & 7.34 & 0.03 & 0.35 & 0.15 \\
\hline F7 & VR c & 0.58 & 3.32 & 1.93 & 8.00 & 0.05 & 0.71 & 0.18 \\
\hline F9 & $V R j$ & 0.61 & 3.47 & 2.11 & 7.42 & 0.05 & 0.97 & 0.11 \\
\hline F10 & Glass k & 0.77 & 3.87 & 2.97 & 11.47 & 0.03 & 0.84 & 0.10 \\
\hline F11 & $\mathrm{V} \mathrm{Rb}$ & 0.80 & 3.48 & 2.78 & 8.41 & 0.04 & 0.64 & 0.17 \\
\hline F16 & Glass C & 0.81 & 3.76 & 3.05 & 11.27 & 0.02 & 0.59 & 0.12 \\
\hline F18 & Glass l & 1.27 & - & - & 16.30 & 0.01 & 0.58 & 0.08 \\
\hline F21 & Glass $\mathrm{T}$ & 0.68 & 3.39 & 2.30 & 7.86 & 0.04 & 0.56 & 0.17 \\
\hline F22 & Glass D & 1.03 & 4.10 & 4.23 & 12.68 & 0.09 & 1.41 & 0.16 \\
\hline F23 & Glass E & 0.90 & 3.97 & 3.57 & 14.34 & 0.09 & 1.52 & 0.14 \\
\hline F24 & HD 96675 & 0.31 & 3.38 & 1.05 & 9.26 & 0.04 & 0.58 & 0.21 \\
\hline F25 & Glass B & 1.27 & 3.82 & 4.85 & 13.37 & 0.02 & 0.65 & 0.13 \\
\hline F28 & Glass $\mathrm{G}$ & 1.10 & - & - & 23.75 & 0.02 & 0.76 & 0.12 \\
\hline F29 & Glass h & 0.31 & 4.06 & 1.26 & 20.37 & 0.01 & 1.04 & 0.09 \\
\hline F30 & Glass A & 0.46 & 2.70 & 1.24 & 11.31 & 0.02 & 0.49 & 0.14 \\
\hline F33 & Glass O & 1.05 & 9.51 & 9.98 & 25.24 & 0.01 & 0.31 & 0.32 \\
\hline F34 & Glass Q & 0.53 & 3.56 & 1.89 & 18.91 & 0.03 & 0.69 & 0.12 \\
\hline F36 & Glass P & 1.22 & 4.79 & 5.85 & 21.19 & 0.02 & 0.87 & 0.11 \\
\hline F37 & $\mathrm{VR} \mathrm{h}$ & 0.31 & 3.41 & 1.06 & 4.99 & 0.08 & 1.00 & 0.18 \\
\hline F39 & VR i & 0.32 & 3.13 & 1.00 & 4.08 & 0.08 & 0.49 & 0.19 \\
\hline F40 & HD 98143 & 0.65 & 3.51 & 2.28 & 12.14 & 0.03 & 0.77 & 0.16 \\
\hline F41 & HD 98294 & 0.26 & 3.42 & 0.89 & 4.10 & 0.12 & 1.02 & 0.17 \\
\hline F42 & HD 98561 & 0.34 & 3.51 & 1.19 & 4.63 & 0.11 & 0.93 & 0.18 \\
\hline F43 & HD 98627 & 0.25 & 4.15 & 1.04 & 2.39 & 0.63 & 2.10 & 0.19 \\
\hline F44 & HD 98672 & - & - & - & 5.60 & 0.08 & 0.85 & 0.24 \\
\hline F47 & HD 99015 & 0.05 & - & - & 2.01 & 0.12 & 0.86 & 0.23 \\
\hline F48 & HD 99161 & 0.18 & 3.41 & 0.61 & 2.20 & 0.08 & 0.59 & 0.16 \\
\hline F50 & HD 99515 & 0.13 & 3.21 & 0.42 & 0.79 & 0.26 & 0.65 & 0.32 \\
\hline F52 & HD 99759 & 0.30 & 3.54 & 1.06 & 5.87 & 0.08 & 0.82 & 0.19 \\
\hline F54 & HD 100401 & 0.28 & 3.24 & 0.91 & 3.86 & 0.13 & 1.14 & 0.18 \\
\hline
\end{tabular}

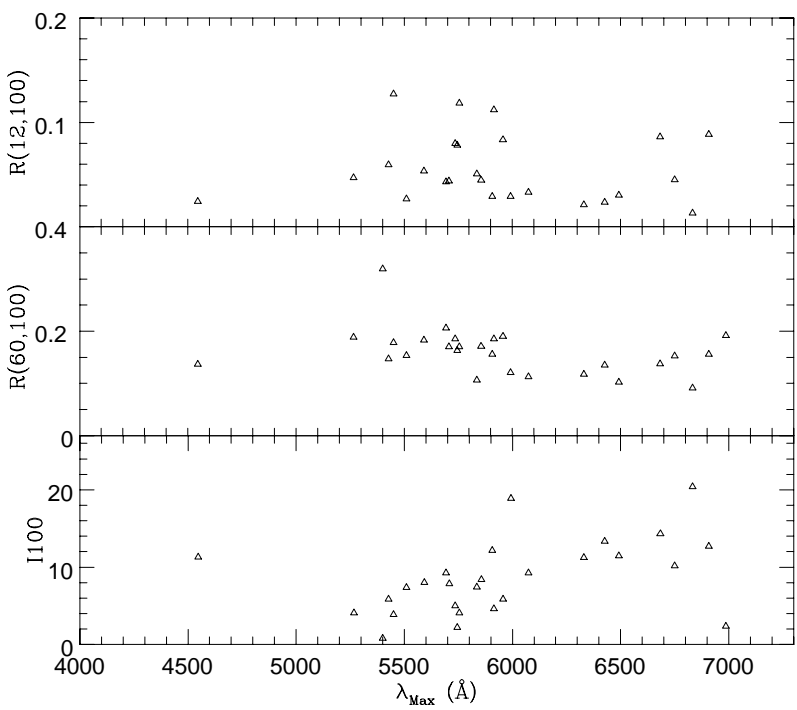

Fig. 9. Infrared color indices versus the wavelength of maximum polarization, $\lambda_{\mathrm{Max}}$. A strong correlation is seen with $I_{100 \mu}$, and a very weak trend is seen between $R(12,100)$ and $\lambda_{\mathrm{Max}}$, suggesting that the small grains responsible for $R(12,100)$ enhancements do not appreciably affect the grain size distribution variations in the far UV rise of the extinction curves for stars in Chamaeleon I and II were not correlated with 12 and $25 \mu \mathrm{m}$ emission, which they interpreted as proof that the $R(12,100)$ fluctuations were caused by slight variations in the size distributions of mid sized and small grains having the same composition as the larger grains. Our results are consistent with this model, since only a slight trend is seen between $R(12,100)$ and $\lambda_{\text {Max }}$ which is plotted in Fig. 9. We do see a strong correlation between $I_{100 \mu}$ and $\lambda_{\text {Max }}$, however, and a slight anticorrelation between $R(60,100)$ and $\lambda_{\mathrm{Max}}$. These results suggest that the larger grains associated with increased values of $\lambda_{\text {Max }}$ also have reduced grain temperatures and larger $100 \mu \mathrm{m}$ fluxes.

We have also made plots of the IRAS fluxes and colours against the maximum percentage polarization $P_{\max }$, and these are presented in Figs. 10a and 10b. A definite correlation between $I_{100 \mu}$ and $P_{\max }$ is seen, as is an anticorrelation between $R(12,100)$ and $P_{\max }$. These results strongly suggest that the larger grains which have increased values of $I_{100}$ and $\lambda_{\text {Max }}$ also are responsible for large amounts of polarization. Colour-colour plots of $R(12,100)$ against $I_{100 \mu}$ (Fig. 11) reinforce the trends shown in Figs. 10a and $10 \mathrm{~b}$, as does the plot of $R(60,100)$ vs. $R(12,100)$ (Fig. 11, bottom), which suggests that the larger values of $R(60,100)$, usually associated with heated grains, also give rise to larger numbers of small particles which are responsible for the increase of $R(12,100)$. 
Table 4. Spectroscopic results

\begin{tabular}{llcccccccc}
\hline \hline$\#$ & Star & $\begin{array}{c}b_{\mathrm{CaII}} \\
\left(\mathrm{km} \mathrm{s}^{-1}\right)\end{array}$ & $\begin{array}{c}N_{\mathrm{CaII}} \\
\left(\mathrm{cm}^{-2}\right)\end{array}$ & $\begin{array}{c}V_{\mathrm{lsr}, \mathrm{CaII}} \\
\left(\mathrm{km} \mathrm{s}^{-1}\right)\end{array}$ & $\begin{array}{c}W_{\mathrm{CaII}} \\
(\AA)\end{array}$ & $\begin{array}{c}b_{\mathrm{CH}} \\
\left(\mathrm{km} \mathrm{s}^{-1}\right)\end{array}$ & $\begin{array}{c}N_{\mathrm{CH}} \\
\left(\mathrm{cm}^{-2}\right)\end{array}$ & $\begin{array}{c}V_{\mathrm{lsr}, \mathrm{CH}} \\
\left(\mathrm{km} \mathrm{s}^{-1}\right)\end{array}$ & $\begin{array}{c}W_{\mathrm{CH}} \\
(\AA)\end{array}$ \\
\hline \hline F2 & VR g & 3.13 & $4.68 \mathrm{E} 11$ & 8.66 & 0.0514 & - & - & - & - \\
F2 & VR g & 3.12 & $1.01 \mathrm{E} 12$ & -0.38 & 0.0724 & - & - & - & - \\
F3 & HD 94414 & 2.10 & $6.85 \mathrm{E} 11$ & 4.80 & 0.0383 & 1.16 & $4.52 \mathrm{E} 13$ & 4.35 & 0.0247 \\
F3 & HD 94414 & 1.80 & $1.55 \mathrm{E} 11$ & -2.88 & 0.0120 & - & - & - & \\
F24 & HD 96675 & 1.90 & $4.03 \mathrm{E} 11$ & 4.58 & 0.0252 & 0.86 & $3.13 \mathrm{E} 13$ & 3.74 & 0.0187 \\
F24 & HD 96675 & 1.80 & $4.9 \mathrm{E} 10$ & -3.72 & 0.0054 & - & - & - & \\
T32 & HD 97048 & 1.67 & $6.67 \mathrm{E} 11$ & -7.751 & 0.0573 & 1.069 & $3.435 \mathrm{E} 13$ & 4.28 & 0.0180 \\
T41 & HD 97300 & 1.51 & $8.11 \mathrm{E} 11$ & 4.16 & 0.0392 & 1.60 & $1.74 \mathrm{E} 13$ & 4.63 & 0.0112 \\
F40 & HD 98143 & 1.90 & $1.02 \mathrm{E} 12$ & 4.65 & 0.0398 & 0.61 & $8.05 \mathrm{E} 12$ & 7.52 & 0.0051 \\
F40 & HD 98143 & 1.80 & $9.95 \mathrm{E} 12$ & -2.88 & 0.0102 & - & - & - & \\
F41 & HD 98294 & 2.09 & $5.61 \mathrm{E} 11$ & 1.81 & 0.0386 & 0.76 & $1.34 \mathrm{E} 13$ & 2.88 & 0.0080 \\
F42 & HD 98561 & - & - & - & - & 0.74 & $1.14 \mathrm{E} 13$ & 2.67 & 0.0068 \\
F48 & HD 99161 & 2.24 & $1.55 \mathrm{E} 12$ & 2.03 & 0.074 & 0.71 & $5.48 \mathrm{E} 12$ & 3.49 & 0.0036 \\
F48 & HD 99161 & 1.05 & $9.59 \mathrm{E} 10$ & -4.52 & 0.0087 & - & - & - & \\
F52 & HD 99759 & 1.55 & $8.46 \mathrm{E} 11$ & 1.72 & 0.051 & 0.90 & $2.98 \mathrm{E} 13$ & 3.18 & 0.0177 \\
F52 & HD 99759 & 2.60 & $1.04 \mathrm{E} 11$ & -2.76 & 0.0077 & - & - & - & \\
\hline \hline
\end{tabular}

Table 4. continued

\begin{tabular}{llccccccrr}
\hline \hline$\#$ & Star & $\begin{array}{c}b_{\mathrm{CaI}} \\
\left(\mathrm{km} \mathrm{s}^{-1}\right)\end{array}$ & $\begin{array}{c}N_{\mathrm{CaI}} \\
\left(\mathrm{cm}^{-2}\right)\end{array}$ & $\begin{array}{c}V_{\mathrm{lsr}, \mathrm{CaI}} \\
\left(\mathrm{km} \mathrm{s}^{-1}\right)\end{array}$ & $\begin{array}{c}W_{\mathrm{CaI}} \\
(\AA)\end{array}$ & $\begin{array}{c}b_{\mathrm{CH}+} \\
\left(\mathrm{km} \mathrm{s}^{-1}\right)\end{array}$ & $\begin{array}{c}N_{\mathrm{CH}+} \\
\left(\mathrm{cm}^{-2}\right)\end{array}$ & $\begin{array}{c}V_{\mathrm{lsr}, \mathrm{CH}+} \\
\left(\mathrm{km} \mathrm{s}^{-1}\right)\end{array}$ & $\begin{array}{c}W_{\mathrm{CH}+} \\
(\AA)\end{array}$ \\
\hline \hline F2 & VR g & - & - & - & - & - & - & - & - \\
F2 & VR g & - & - & - & - & - & - & - & - \\
F24 & HD 96675 & - & $<4.71 \mathrm{E} 09$ & - & $<0.0013$ & - & $<3.20 \mathrm{E} 12$ & - & $<0.0028$ \\
T32 & HD 97048 & - & $<1.95 \mathrm{E} 09$ & - & $<0.0036$ & - & $<1.50 \mathrm{E} 12$ & - & $<0.0013$ \\
T41 & HD 97300 & - & $<1.42 \mathrm{E} 09$ & - & $<0.0026$ & 1.06 & $1.46 \mathrm{E} 11$ & 0.84 & 0.0001 \\
T41 & HD 97300 & - & - & - & - & 1.04 & $3.34 \mathrm{E} 12$ & 2.59 & 0.0030 \\
F40 & HD 98143 & - & $<2.90 \mathrm{E} 10$ & - & $<0.0080$ & - & $<7.65 \mathrm{E} 12$ & - & $<0.0068$ \\
F41 & HD 98294 & - & $<1.46 \mathrm{E} 10$ & - & $<0.0040$ & - & $<6.83 \mathrm{E} 12$ & - & $<0.0060$ \\
F42 & HD 98561 & - & - & - & - & - & - & - & - \\
F48 & HD 99161 & - & $<1.70 \mathrm{E} 10$ & - & $<0.0047$ & - & $<1.38 \mathrm{E} 13$ & - \\
F52 & HD 99759 & - & $<1.17 \mathrm{E} 10$ & - & $<0.0032$ & 2.00 & $1.88 \mathrm{E} 13$ & 0.31 \\
\hline \hline
\end{tabular}

\subsection{Spectroscopic results}

The transitions from large to small grains are generally attributed to variations in either the ambient UV radiation field or convective turbulent motions within the interstellar cloud. Both processes would be expected to affect the depletion of ices from the surfaces of grains, and to release highly depleted elements such as $\mathrm{Ca}$ into the gas phase. The observation of $\mathrm{Ca}$ II in absorption from background stars should therefore provide a useful probe of grain destruction or ablation from radiation or kinetic processes. We present in Fig. 12 the absorption lines of Ca II for our sample, in order of Right Ascension of the star. Line profile models for all the spectra have been computed, and the values of $v_{\mathrm{lsr}}, b$, and $N(\mathrm{Ca} \mathrm{II})$ are presented in Table
4. The Ca II absorption is quite uniform across the cloud in velocity, although in some sight lines a weak second component of absorption appears. The second component is strongest in the spectra at either edge of the Cha I cloud, suggesting that this component may arise from either a neighboring cloud, or higher velocity gas which is impacting the Cha I region.

High resolution spectra of $\mathrm{CH}$ absorption are presented for the sample of stars in Fig. 13. The fitted line profiles are included, and the values of $v_{\mathrm{lsr}}, b$, and $N(\mathrm{CH})$ are also presented in Table 4 . The molecular content of clouds is generally found to correlate with $100 \mu \mathrm{m}$ emission, since the larger grains which emit $100 \mu \mathrm{m}$ are effective in shielding the molecules from the dissociating interstellar radiation 


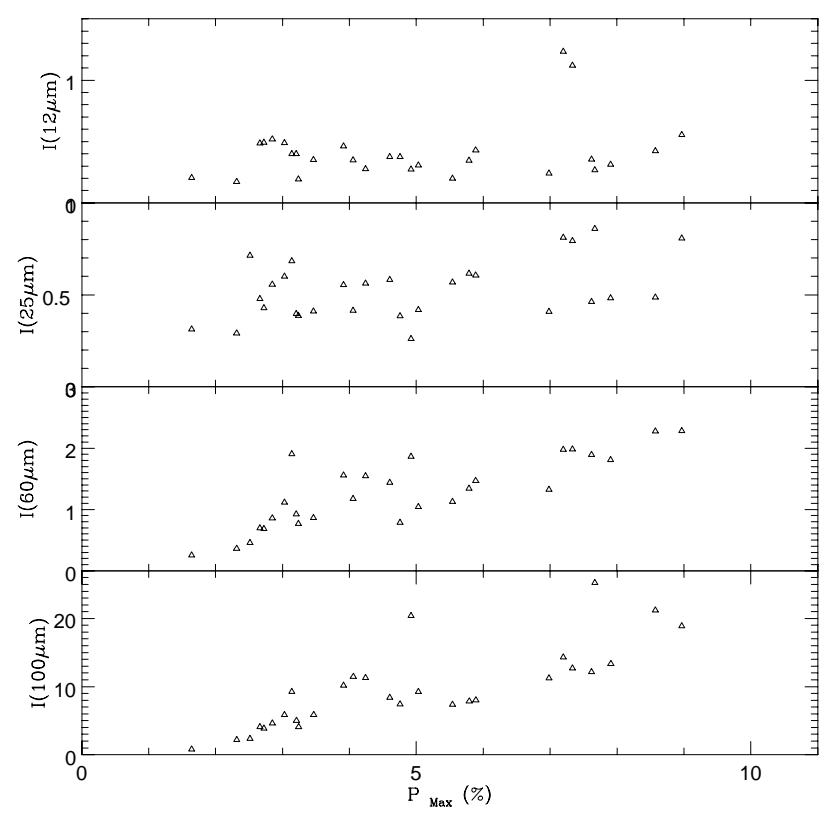

Fig. 10. a) Plots of IRAS fluxes against $P_{\text {Max }}$, the maximum polarization percentage from Serkowski fit. Strong correlations are seen in the 60 and $100 \mu \mathrm{m}$ fluxes

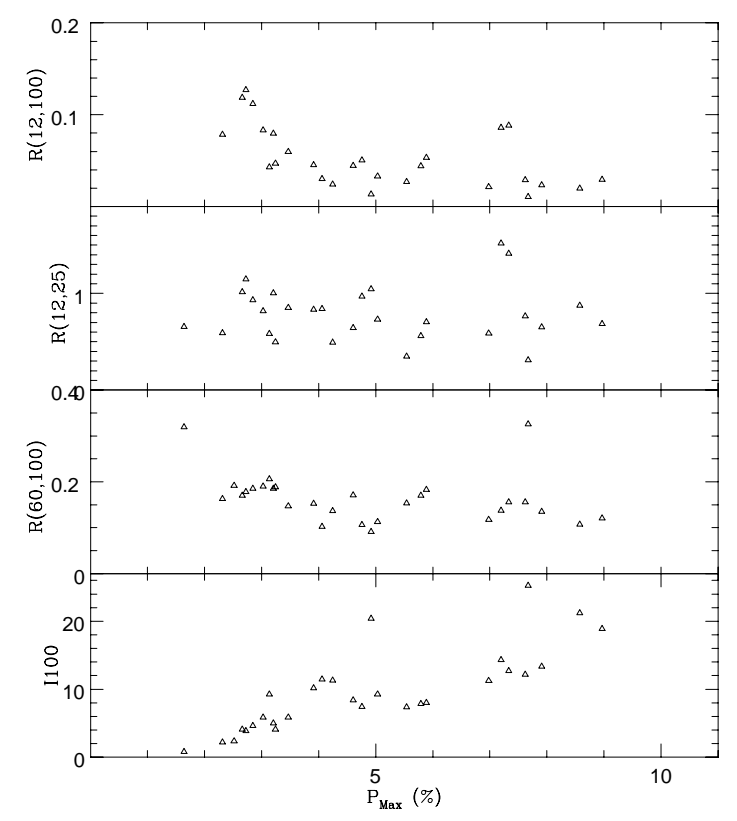

Fig. 10. b) Plots of IRAS colors against $P_{\text {Max }}$, the maximum polarization percentage from Serkowski fit. Strong anti-correlations are seen in the $R(60,100)$ and $R(12,100)$ plots, suggesting that sightlines with grain heating also have substantial dealignment of the polarizing grains
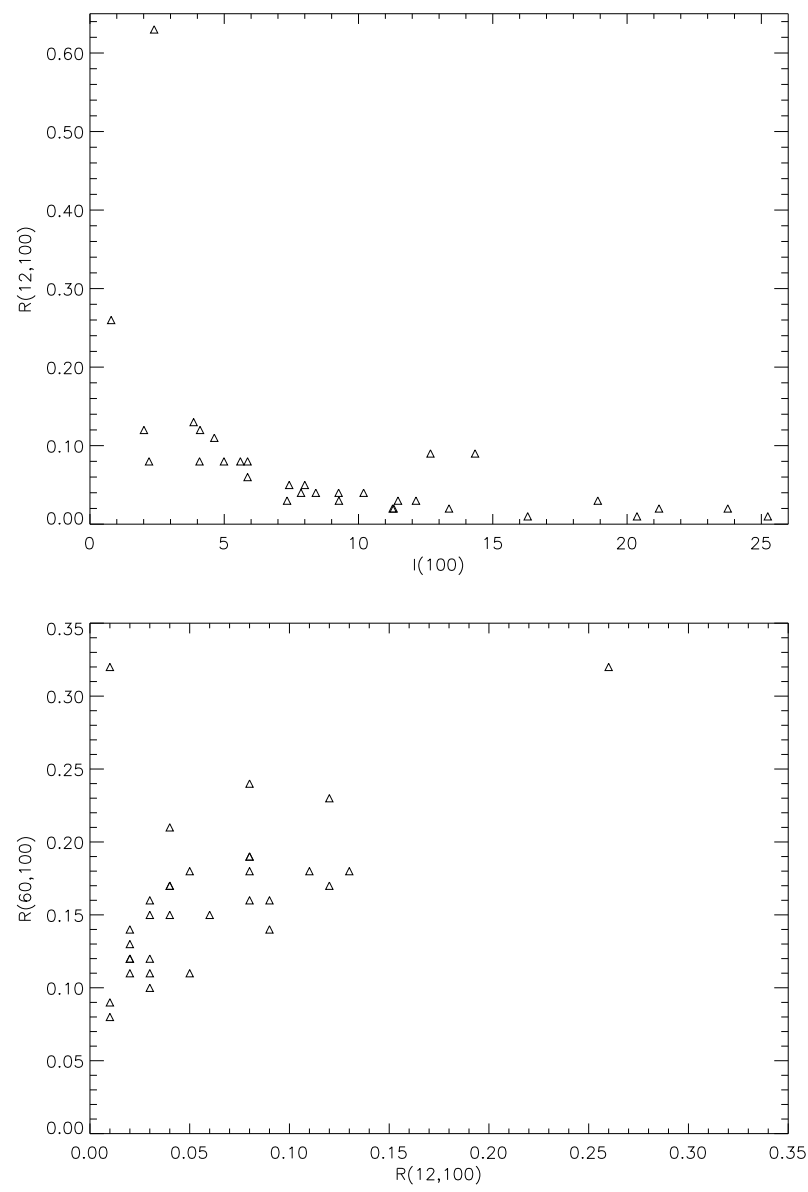

Fig. 11. Color-color IRAS plot for the points on the Cha I cloud toward stars in our polarimetric sample. (Top): $R(12,100)$ values are seen to highest where $I_{100 \mu}$ is minimized, which is consistent with an interpretation of $R(12,100)$ enhancements as heating at the edge of the Cha I cloud. (Bottom): $R(60,100)$ values are seen to correlate with $R(12,100)$, as is seen in other diffuse clouds

field. However, if transient processes exist in molecular clouds which heat small grains and can produce molecular $\mathrm{CH}$, then it should be possible to see trends between $N(\mathrm{CH})$ with $E_{B-V}$ and $R(60,100)$. Recent observations have found significant amounts of $\mathrm{CH}$ in the warm envelopes of molecular clouds, suggesting that both $\mathrm{CH}$ and $\mathrm{CH}^{+}$may exist as a transient phase of molecular gas in some molecular clouds (Crane et al. 1995).

Figures 14a-d present stacked spectra for single sight lines, and it is clear that the $\mathrm{CH} / \mathrm{CH}^{+}$ratio is highly variable in the Cha I cloud. In the sections below, we discuss the data for each of the four Cha I sight lines, detailing the relationships between IRAS and spectroscopic results.

\subsubsection{HD 99759}

Figure 14a shows a stacked plot of the spectra for the HD 99759 sight line, which appears toward the Eastern 


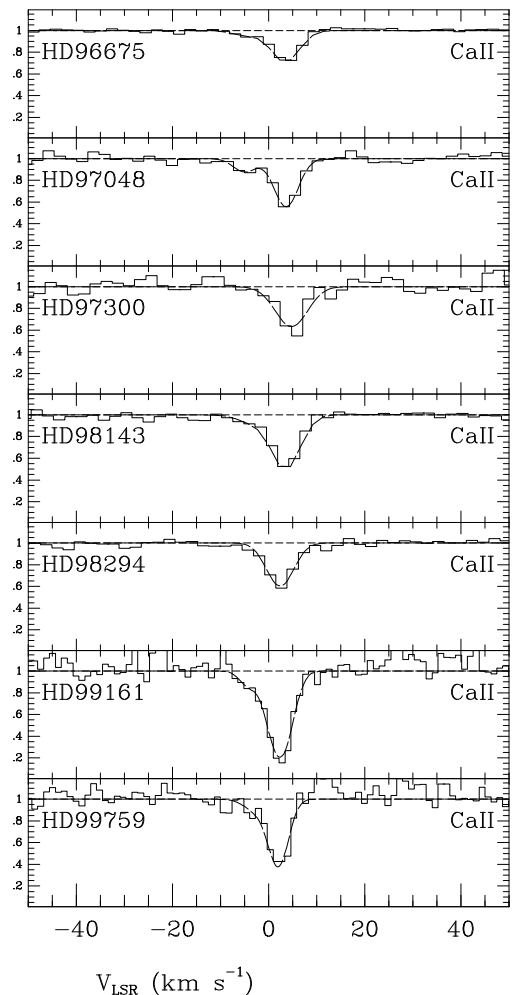

Fig. 12. a) Spectra for all of our spectroscopic program stars in Ca II absorption, in order of right ascension. The Cha I cloud Ca II absorption appears at a nearly constant velocity of $v_{\mathrm{lsr}}=4.5 \mathrm{~km} \mathrm{~s}^{-1}$, with a second blended component visible in sightlines at either end of the cloud. The strongest Ca II absorption appears at the East end of the cloud

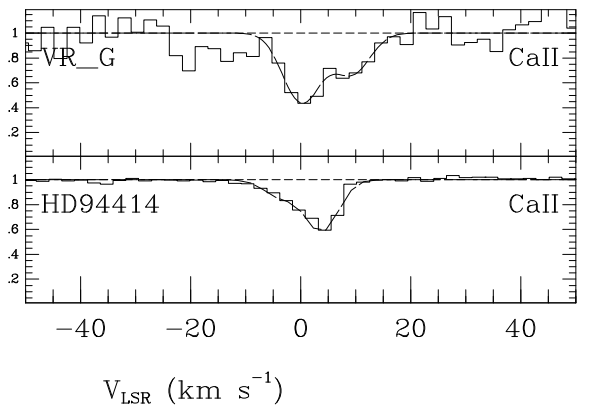

Fig. 12. b)

edge of the Cha I cloud, where the IRAS $100 \mu \mathrm{m}$ flux is $I_{100 \mu}=5.87 \mathrm{mJy} \mathrm{Sr}^{-1}$. The observed Ca II absorption profile has been fitted by two components giving a total column density $N(\mathrm{Ca}$ II $)=8.510^{11} \mathrm{~cm}^{-2}$. The molecular content for this sight line is high with $N(\mathrm{CH})=3.0$ $10^{13} \mathrm{~cm}^{-2}$ and $N\left(\mathrm{CH}^{+}\right)=1.910^{13} \mathrm{~cm}^{-2}$. The $\mathrm{CH}^{+}$profile was fitted by a single component and appears asymmetric, which may be the result of two blended components.

The extinction for the sight line is only $E_{B-V}=0.30$, and the column density of $\mathrm{CH}$ is one of the highest per

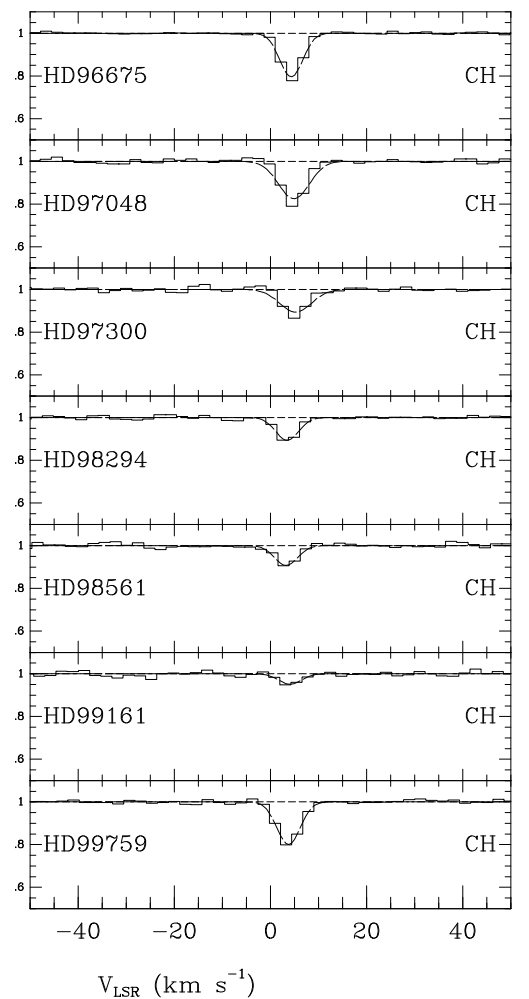

Fig. 13. a) Spectra for all of our spectroscopic program stars in CH II absorption, in order of right ascension. The Cha I cloud CH II absorption appears in a single strong component at the same velocity of the dominant Ca II absorption line. The strongest $\mathrm{CH}$ absorption appears at either end of the cloud

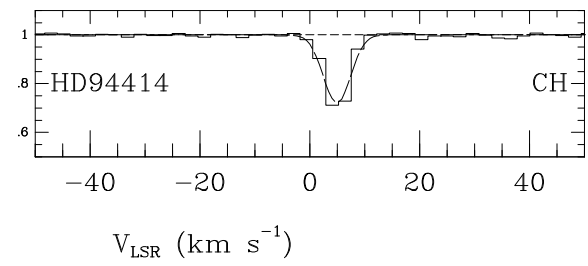

Fig. 13. b)

unit of extinction, as seen in Fig. 15. In low extinction sight lines, like the one toward HD 99759, the dissociation rate of $\mathrm{CH}$ from the interstellar radiation field must be balanced by an increased production of $\mathrm{CH}$ to account for the large molecular column densities. A production mechanism which could be attributed to shocks which collide with the Cha I cloud would be most pronounced near the edges of the dark cloud region, such as the material toward HD 99759. The large value of $N\left(\mathrm{CH}^{+}\right) / N(\mathrm{CH})=0.63$ is consistent with this hypothesis, since $\mathrm{CH}^{+}$has been thought to be produced in shocks (Allen 1994).

\subsubsection{HD 97048}

Figure 14b shows a stacked plot of the spectra for the HD 97048 sight line, which appears toward the Southern 


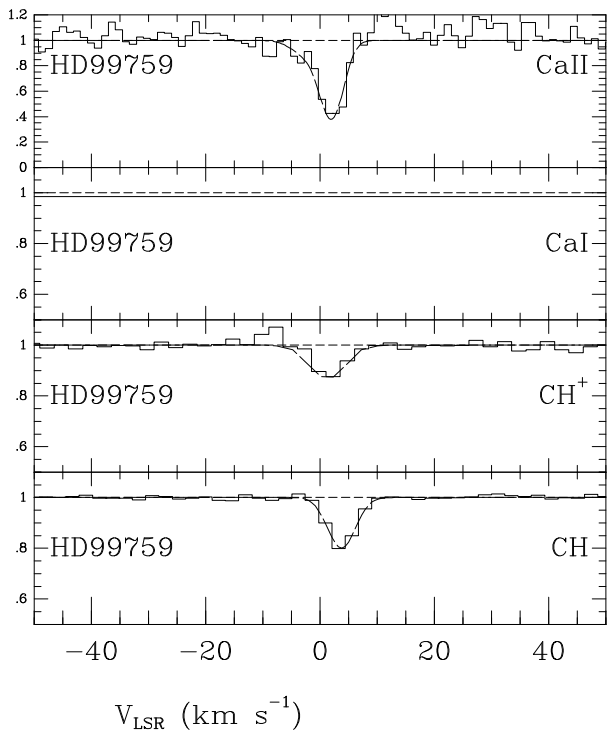

Fig. 14. a) Stacked spectra for the HD 99759 sight line. The absorption lines are discussed in Sect. 3.3.1.

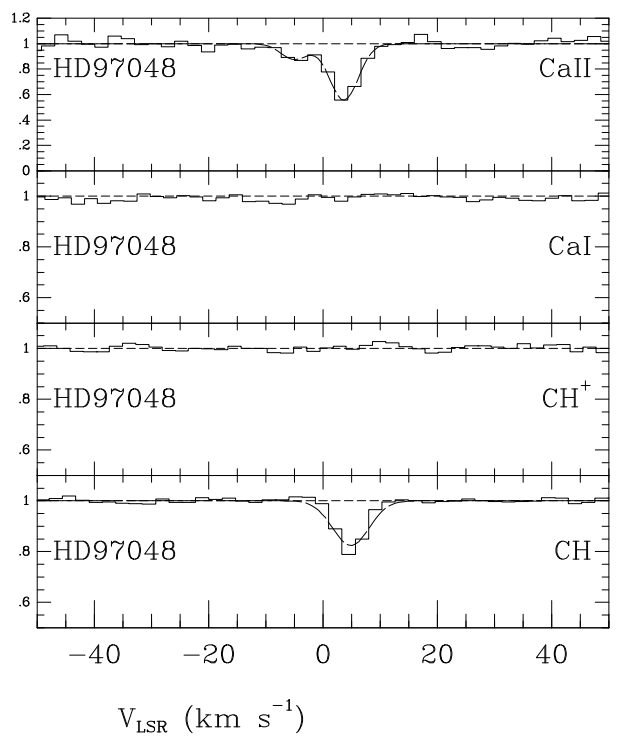

Fig. 14. b) Stacked spectra for the HD 97048 sight line. The absorption lines are discussed in Sect. 3.3.2.

central part of the Cha I cloud, where the IRAS $100 \mu \mathrm{m}$ flux is $I_{100 \mu}=250.1 \mathrm{mJy} \mathrm{Sr}^{-1}$. HD 97048 is thought to be embedded in the Cha I cloud, and the large IRAS fluxes therefore come from circumstellar material being heated by the star. HD 97048 is associated with the reflection nebula Ced 111, and has been found to be surrounded by additional IRAS sources, which suggests that HD 97048 is a center for low-mass star formation. Recent reviews on the HD 97048 and HD 97300 sight lines include Assendorp et al. (1990), and Steenman \& Thé (1989). The latter have found that the two stars have anomalous extinction, with $R_{V}=5.0$, which would result in an optical extinction for

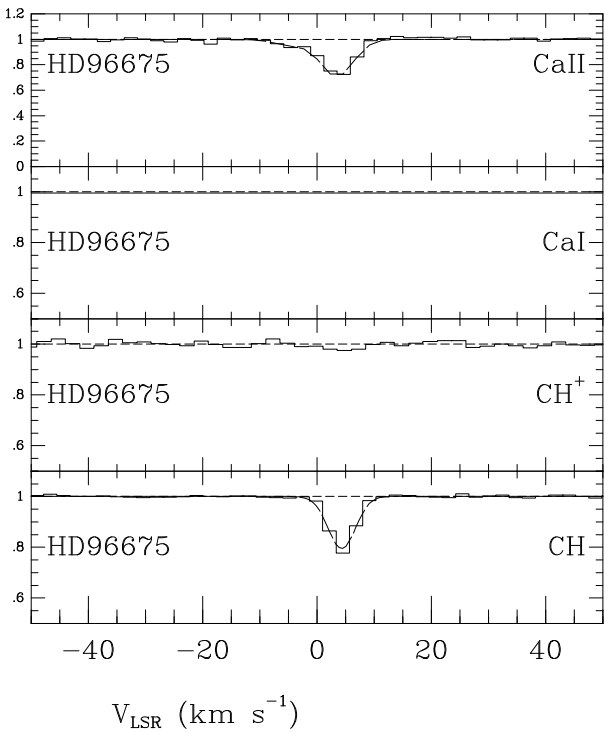

Fig. 14. c) Stacked spectra for the HD 96675 sight line. The absorption lines are discussed in Sect. 3.3.3.

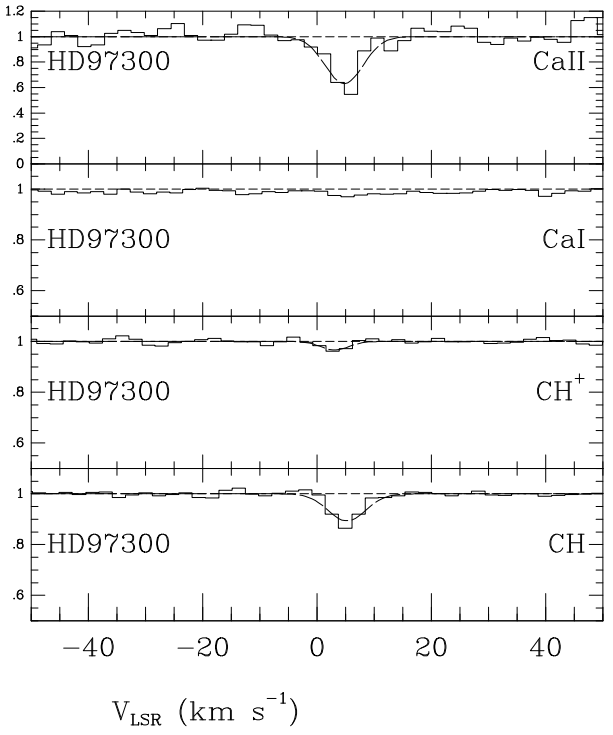

Fig. 14. d) Stacked spectra for the HD 97300 sight line. The absorption lines are discussed in Sect. 3.3.4.

HD 97048 of $A_{V}=1.75$, which is less than half of the estimated maximum extinction of $A_{V}=5.0$ for the Cha I cloud. The HD 97048 sight line would therefore be on the near side of the Cha I cloud, within a reflection nebula which consists of a mixture of radiation processed gas and dust. The $\mathrm{CH}$ column density for HD 97048 is slightly higher than the HD 99759, at $N(\mathrm{CH})=3.410^{13} \mathrm{~cm}^{-2}$, which is surprising considering the large radiation field which must be present from the embedded source HD 97048. The $\mathrm{CH}^{+}$column density for this sight line is extremely low, with $N\left(\mathrm{CH}^{+}\right)<1.510^{12} \mathrm{~cm}^{-2}$. 


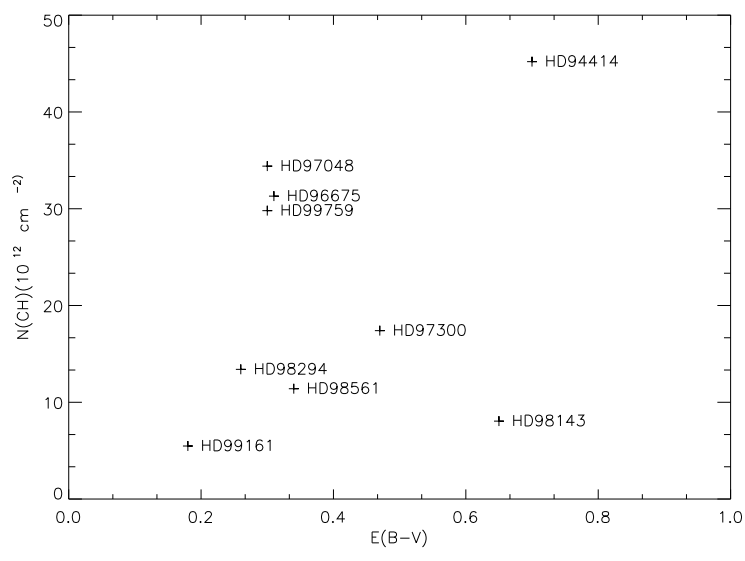

Fig. 15. $\mathrm{CH}$ column density, $N(\mathrm{CH})$, is plotted against $E_{B-V}$ for spectroscopic program stars, showing enhanced $N(\mathrm{CH}) / E_{B-V}$ content for sightlines toward HD 94414, HD 97300, HD 96675, and HD 99759

It is interesting to note that two sight lines from the same cloud, HD 97048 and HD 99759, have nearly identical colour excesses of $E_{B-V}=0.3$, and yet have extremely different ratios of $N\left(\mathrm{CH}^{+}\right) / N(\mathrm{CH})$, with value of $N\left(\mathrm{CH}^{+}\right) / N(\mathrm{CH})<0.043$ for $\mathrm{HD} 97048$ and $N\left(\mathrm{CH}^{+}\right) / N(\mathrm{CH})=0.63$ for HD 99759 , respectively. This difference may be due to the fact that HD 99759 is preferentially sampling the edge of the cloud, where our results strongly suggest $\mathrm{CH}^{+}$production is enhanced.

The Ca II column density toward HD 97048 is large, with $N($ Ca II $)=6.710^{11} \mathrm{~cm}^{-2}$, from a single component of absorption. The HD 97048 Ca II absorption is more symmetric than the HD $99759 \mathrm{Ca}$ II absorption, which is also consistent with the enhanced $\mathrm{CH}^{+}$production toward HD 99759 resulting from evaporation or shock processing of gas at the edge of the Cha I dark cloud, which might introduce the extra component of Ca II absorption.

\subsubsection{HD 96675}

Figure 14c shows a stacked plot of the spectra for the HD 96675 sight line, which appears toward a Northern section of the Cha I cloud, where the IRAS $100 \mu \mathrm{m}$ flux is $I_{100 \mu}=9.2 \mathrm{mJy} \mathrm{Sr}^{-1}$. The Ca II column density toward HD 96675 is moderate, with $N(\mathrm{Ca}$ II $)=4.510^{11} \mathrm{~cm}^{-2}$, distributed over two components as for HD 99759. The extinction is again similar to that of the previous two sight lines, with $E_{B-V}=0.31$, and with a substantial $\mathrm{CH}$ column density of $N(\mathrm{CH})=3.110^{13} \mathrm{~cm}^{-2}$, one of the highest per unit of extinction, as seen in Fig. 15. The $\mathrm{CH}^{+}$column density is very weak, with $N\left(\mathrm{CH}^{+}\right)<3.210^{12} \mathrm{~cm}^{-2}$. It turns out that the HD 96675 sight line has a large molecular content, yet a small ratio of $N\left(\mathrm{CH}^{+}\right) / N(\mathrm{CH})$ and $N(\mathrm{Ca}$ II $) / N(\mathrm{CH})$. The high value of $I_{100 \mu} / E_{B-V}$ may hint at a different value of $R_{V}$ for this sight line, or may reflect a substantial population of large grains which would shield the $\mathrm{CH}$ from dissociation, but perhaps provide a less favourable environment for the production of $\mathrm{CH}^{+}$. The value of the total to selective extinction ratio calculated from the wavelength dependence of the polarization is $R_{V}=3.3$, although the value of $\lambda_{\max }$ suggested from the polarimetry is $\lambda_{\max }=5700 \AA$, which is average for the polarimetry sample. The IRAS color index $R(12,100)=0.04$ is very small, suggesting a reduced population of heated small grains, and the value of $R(60,100)=0.21$ corresponds to one of the lowest grain temperatures of the sample.

\subsubsection{HD 97300}

Figure 14d shows a stacked plot of the spectra for the HD 97300 sight line, which appears slightly South of HD 96675 but still in the central part of the cloud. Like HD 97048, HD 97300 is believed to be embedded in the Cha I dark cloud, and therefore the IRAS colours are from heated circumstellar material. The IRAS $100 \mu \mathrm{m}$ flux is reported to be $I_{100 \mu}=282 \mathrm{mJy} \mathrm{Sr}^{-1}$. The $\mathrm{CH}^{+}$column density for this sight line is higher than for HD 97048, with $N\left(\mathrm{CH}^{+}\right)=3.310^{12} \mathrm{~cm}^{-2}$, and $N\left(\mathrm{CH}^{+}\right) / N(\mathrm{CH})=0.08$, which is at least twice as high as the HD 97048 sight line, but still relatively low compared with other molecular absorption sight lines. The Ca II column density is substantially higher than for the HD 97048 sight line, with $N(\mathrm{Ca} \mathrm{II})=8.110^{11} \mathrm{~cm}^{-2}$. The column densities of $\mathrm{CH}^{+}$ and Ca II therefore seem to be correlated, at least for our limited spectroscopic sample, which again suggests that the $\mathrm{CH}^{+}$appears in the warmer outer envelopes of molecular clouds.

\subsubsection{Variation of Column Densities with $E_{B-V}$ and IRAS colours}

Figure 15 is a plot of $N(\mathrm{CH})$ vs. $E_{B-V}$ and we see a definite increase in $N(\mathrm{CH})$ with increased extinction. Also notable is the very large value of $N(\mathrm{CH}) / E_{B-V}$ for the stars HD 94414, HD 97300, HD 96675, and HD 99759. Variations in $N(\mathrm{CH}) / E_{B-V}$ have been observed previously in high galactic latitude molecular clouds and may trace shock formation of molecules (Penprase 1993; Penprase et al. 1990).

One of the more interesting results from the comparison of spectroscopic, polarimetric and IRAS data involved the differing behaviour of $N(\mathrm{Ca}$ II $)$ and $N(\mathrm{CH})$ with IRAS colours and $\lambda_{\max }$. Figure 16 presents a plot of the column densities $N(\mathrm{CH})$ and $N(\mathrm{Ca}$ II) against $R(60,100)$, which is commonly considered to be a good indicator of grain temperature. Values of $N(\mathrm{CH})$ are seen to rise steadily with $R(60,100)$, suggesting strongly that the warmer grains are somehow producing additional $\mathrm{CH}$. At the same time, Ca II appears to decrease with grain temperature, which is surprising since much of the gas phase Ca II is expected to result from the disruption (and therefore heating) of grains at the edges of clouds. 

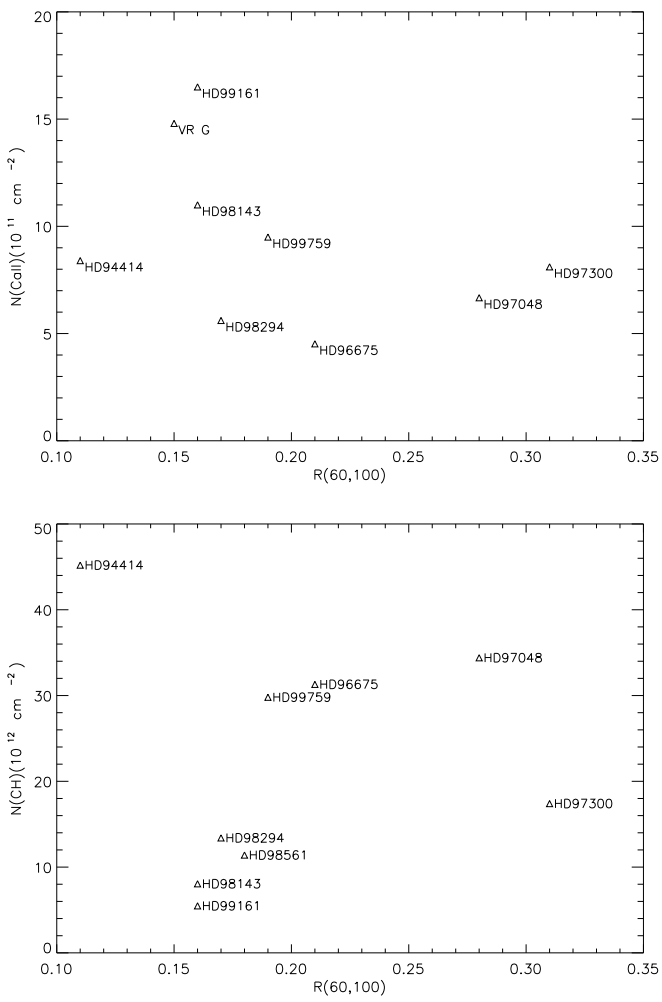

Fig. 16. Plots of $N(\mathrm{Ca} \mathrm{II})$ (top) and $N(\mathrm{CH})$ (bottom) against $R(60,100)$, commonly considered as a diagnostic of dust temperature. An increasing $\mathrm{CH}$ column density is seen toward the warmer sightlines, while Ca II content decreases

Another interesting relation is found between $N(\mathrm{CH}) / I_{100 \mu}$ and $\lambda_{\max }$, which is shown in Fig. 17. $N(\mathrm{CH}) / I_{100 \mu}$ decreases with $\lambda_{\max }$, while $N(\mathrm{Ca}$ II $) / I_{100 \mu}$ is unchanged. One interpretation of this result is that the $\mathrm{CH}$ production may be more favourable on smaller grains, which have decreased values of $\lambda_{\max }$. Further observations are needed to test this possibility.

\section{Conclusions and summary}

We have found many consistent relationships between the grain and gas phase diagnostics of the interstellar medium of the Cha I cloud. The warmer interstellar material, based on the IRAS colour index $R(60,100)$ has more molecular $\mathrm{CH}$, while $\mathrm{CH}^{+}$shows up toward sight lines at cloud edges with enhanced values of $R(12,100)$ and strong $\mathrm{Ca}$ II absorption. The large column densities of $\mathrm{CH}$ and the high values of $N(\mathrm{CH}) / E_{B-V}$ for the stars in our sample suggest a possible additional production mechanism of $\mathrm{CH}$ which outpaces the photodissociation of $\mathrm{CH}$ at cloud edges. The Ca II line profiles toward Cha I show a consistent velocity structure, where a single strong component at $v_{\mathrm{LSR}}=4.5 \mathrm{~km} \mathrm{~s}^{-1}$ is seen along all sight lines. Several sight lines exhibit a weak component of Ca II at $v_{\mathrm{LSR}}=-1.0 \mathrm{~km} \mathrm{~s}^{-1}$. Our observations suggest that the Ca II absorption lines trace a single dense cloud and an
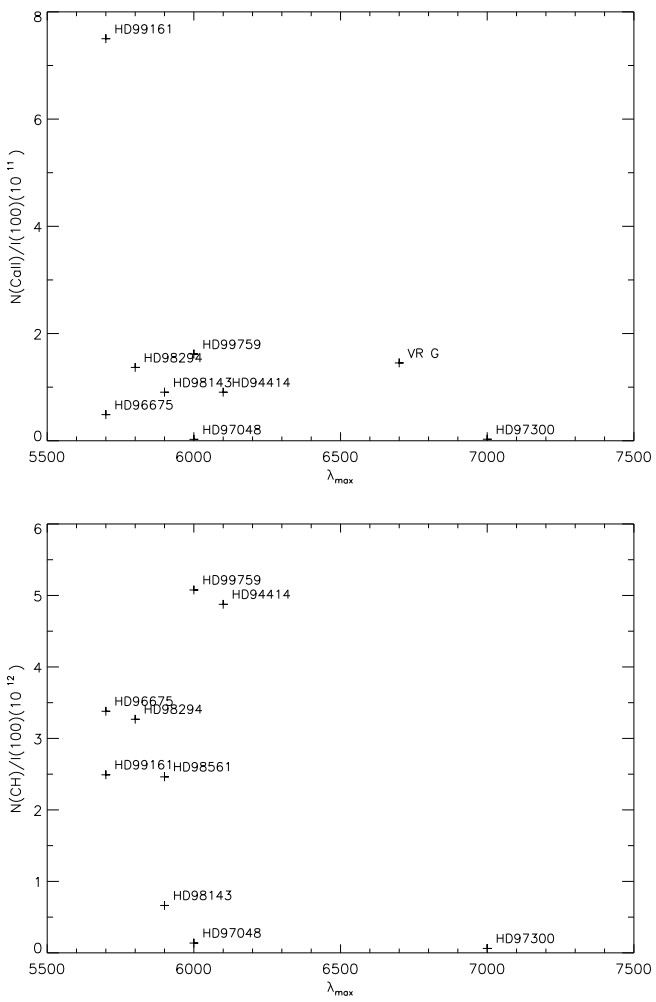

Fig. 17. Plots of normalized densities $N(\mathrm{CH}) / I_{100 \mu}$ and $N(\mathrm{Ca}$ II $) / I_{100 \mu}$ against $\lambda_{\mathrm{Max}}$, derived from the polarimetry. The $\lambda_{\mathrm{Max}}$, values are considered an indicator of grain sizes, and therefore it appears that $\mathrm{CH}$ is produced more rapidly in regions with smaller mean grain sizes

additional warmer cloudlet with a different velocity. The detections of the strongest $\mathrm{CH}^{+}$absorptions appear in sight lines which have the additional Ca II component, suggesting that a possible kinematic disruption of the Cha I cloud plays an important role in $\mathrm{CH}^{+}$production.

Additionally, we observed that the polarization of the stars was less efficient at higher extinctions with $E_{B-V}>0.5$, which we attribute to either more spherical dust grains or increased de-alignment of the grains in the cores of the dense, high extinction sight lines. The IR diagnostics of $R(12,100)$ and $R(60,100)$ were consistent with our observations of polarization and molecular absorption. We found a strong anticorrelation between $R(12,100)$ and the maximum percentage of polarization $P_{\mathrm{Max}}$, suggesting that the processes responsible for the production of the small grain population with strong $12 \mu \mathrm{m}$ emission is also responsible for disrupting the alignment of the grains in the clouds.

A lack of correlation between $R(12,100)$ and $\lambda_{\max }$ suggests that if small grains are responsible for the enhanced values of $R(12,100)$, they are a population which is separate from those responsible for most of the polarization of starlight. If the small grains do arise from the larger grains of the Cha I sight lines as Boulanger \& Gry (1994) have 
suggested, they must evaporate or split from the larger polarizing grains non-destructively in order to maintain the size distributions which indicate large average grain sizes.

Acknowledgements. We wish to thank the European Southern Observatory for the allocation of time for this project. B.E.P. wishes to thank Pomona College for research grants which enabled trips to Europe to collect and analyze data. B.E.P. and E.P. would like to thank the Osservatorio Astronomico di Capodimonte for their hospitality. This research made use of the SIMBAD database, operated at CDS, Strasbourg, France.

\section{References}

Allen M., 1994, ApJ 424, 754

Assendorp R., Wesselius P.R., Whittet D.C.B., Prusti T., 1990, MNRAS 247, 624

Bernard J.P., Boulanger F., 1993, A\&A 277, 609

Boulanger F., Prévot M.L., Gry C., 1994, A\&A 284, 956

Clarke D., Al-Roubaie A., 1984, MNRAS 206, 729

Clarke D., Stewart B.G., 1986, Vistas Astron. 29, 27

Coyne G.V., Gehrels S.J.T., Serkowski K., 1974, AJ 79, 58

Crane P., Lambert D.L., Sheffer Y., 1995, ApJS 99, 107

Dubath P., Reipurth B., Mayor M., 1995, A\&A (in press)

Désert F.X., Boulanger F., Puget J.L., 1990, A\&A 237, 215

Draine B.T., Katz N., 1986, ApJ 306, 655

Feigelson E.D., Casanova S., Montmerle T., Guibert J., 1993, ApJ 416, 623

Franco G.A.P., 1991, A\&A 251, 581

Gauvin L.S., Strom K.M., 1992, ApJ 385, 217

Glass I.S., 1979, MNRAS 187, 305

Grasdalen G., Joyce R., Knacke R.F., Strom S.E., Strom K.M., 1975, AJ 80, 117

Hartigan P., 1993, AJ 105, 1511

Henize K.G., 1954, ApJ 119, 459
Hsu J.-C., Breger M., 1982, ApJ 262, 732

Jones T.J., Hyland A.R., Harvey P.M., Wilking B.A., Joy M., 1985, AJ 90, 1191

McGregor P.J., Harrison T.E., Hough J.H., Bailey J.A., 1994, MNRAS 267, 755

Penprase B.E., Blades J.C., Danks A.C., Crane P., 1990, ApJ 365,241

Penprase B.E., 1993, ApJS 88, 433

Prusti T., Natta A., Palla F., 1994, A\&A 292, 593

Prusti T., Whittet D.C.B., Wesselius P.R., 1991, MNRAS 249, 319

Serkowski K., 1974, in Planets, Stars and Nebulae Studied with Photopolarimetry, Gehrels T. (ed.). Tucson: University of Arizona, p. 135

Schwartz R.D., 1991, in Low Mass Star Formation in Southern Molecular Clouds ESO Scientific Report No. 11, Bo Reipurth (ed.) p. 93

Schwarz H.E., 1989, ESO Operating Manual No. 13

Spitzer L., 1978, Physical Processes in the Interstellar Medium. New York, Wiley, p. 171

Stahl O., Buzzoni B., Kraus G., et al., 1986, ESO Messenger 46,23

Steenman H., Thé P.S., 1989, Ap\&SS 161, 99

Thé P.S., Wesselis P.R., Tjin-A-Djie H.R.E., Steenman H., 1986, A\&A 155, 347

Toriseva M., Mattila K., 1985, A\&A 153, 207

Vrba F.J., Rydgren A.E., 1984, ApJ 283, 123

Walborn N.R., 1968, PASP 80, 162

Whittet D.C.B., Kirrane T.M., Kilkenny D., Oates A.P., Watson F.G., King D.J., 1987, MNRAS 224, 497

Whittet D.C.B., Assendorp R., Prusti T., Roth M., Wesselius P.R., 1991, MNRAS 251, 524

Whittet D.C.B., Gerakines P.A., Carkner A.L., et al., 1994 MNRAS 268, 1,

Zinnecker, et al., 1996 (in preparation) 